\title{
Stability and Hopf Bifurcation Analysis of a Vector-Borne Disease Model with Two Delays and Reinfection
}

\author{
Yanxia Zhang $\mathbb{D}^{1}{ }^{1}$ Long $\mathrm{Li}^{1}{ }^{1}$ Junjian Huang, ${ }^{2}$ and Yanjun $\mathrm{Liu}^{3}$ \\ ${ }^{1}$ School of Mathematics and Information Engineering, Chongqing University of Education, Chongqing 400065, China \\ ${ }^{2}$ Chongqing Key Laboratory of Nonlinear Circuits and Intelligent Information Processing, College of Electronic and \\ Information Engineering, Southwest University, Chongqing 400715, China \\ ${ }^{3}$ School of Mathematical Sciences, Chongqing Normal University, Chongqing 401131, China
}

Correspondence should be addressed to Yanxia Zhang; zhangyx@cque.edu.cn

Received 30 November 2020; Revised 9 May 2021; Accepted 20 May 2021; Published 8 June 2021

Academic Editor: Alireza Jolfaei

Copyright (c) 2021 Yanxia Zhang et al. This is an open access article distributed under the Creative Commons Attribution License, which permits unrestricted use, distribution, and reproduction in any medium, provided the original work is properly cited.

In this paper, a vector-borne disease model with two delays and reinfection is established and considered. First of all, the existence of the equilibrium of the system, under different cases of two delays, is discussed through analyzing the corresponding characteristic equation of the linear system. Some conditions that the system undergoes Hopf bifurcation at the endemic equilibrium are obtained. Furthermore, by employing the normal form method and the center manifold theorem for delay differential equations, some explicit formulas used to describe the properties of bifurcating periodic solutions are derived. Finally, the numerical examples and simulations are presented to verify our theoretical conclusions. Meanwhile, the influences of the degree of partial protection for recovered people acquired by a primary infection on the endemic equilibrium and the critical values of the two delays are analyzed.

\section{Introduction}

Vector-borne diseases (VBDs) are one of the complex infectious diseases that endanger human beings. Vectors are living biological agents, such as ticks, mosquitoes, and fleas, with the ability to transmit parasites, bacteria, or viruses between people or from animals to people. It is reported that VBDs cause more than 1 billion infections and 1 million deaths worldwide every year [1]. Malaria is the most prevalent parasitic vectorborne disease caused by plasmodium parasitizing the human body [2-5]. Plasmodium enters stem cells through the blood to parasitize and reproduce. It then invades red blood cells to reproduce after maturity, causing red blood cells to burst in batches and attack. The source of malaria infection is malaria patients and those with Plasmodium. The natural transmission medium of malaria is female mosquitoes of the genus Anopheles, which is transmitted by biting the human body, and a few by blood transfusion and vertical transmission, with Anopheles gambiae being a major carrier of the disease $[2,5$, 6]. Different types of malaria have different incubation periods, some of which are about 7-12 days, and some of which are more than 6 months. The morbidity and mortality of malaria are high [7]. According to the latest WHO reports, in 2018, there were estimated 228 million malaria cases worldwide, of which the death toll was about 405,000 . It has led to great global economic and social losses, especially in the tropical and subtropical regions on five continents [3].

The population is generally susceptible to malaria. Although there is a certain degree of immunity after several infections, its acquisition process is so slow that it may take years or decades to develop, and it gradually weakens over time $[5,7,8]$. The remaining plasmodium may escape from the immune function due to the antigenic variation and reproduce again (recrudescence); even if the parasite has been eliminated by human immunity or drugs after the initial onset of malaria, the possibility of relapse is not excluded with time. That is, reinfection likely occurs [6,7]. It is shown that time delay is of great significance in many biological modelling, and its change may affect the dynamic behavior of the system $[4-6,9-19]$, such as stability, uniqueness, and 
oscillation of solution. So, it is instructive to consider a mathematical model with time delays to research the influence of immunity on disease control of malaria transmission.

Considering the incubation period of virus transmission in the vector population, some vector-borne epidemic models with a delay were considered [4-6]. In [5], Xu and Zhou proposed a delayed vector-borne epidemic model and reinfection, investigated its existence and stability of equilibrium, and analyzed its dynamical behavior. It suggested that there are two effective preventive measures to reduce infections: one is to minimize vector to human contacts and the other is to use insecticides to control vector. According to $[4,5]$, a vector-borne disease model with delay-saturated infection rate and cure rate was given by [6]. The existence and local stability of the epidemic equilibrium were discussed, and the length of the delay of the system preserving stability was estimated. This paper develops an improved vector-borne disease model with two delays and reinfection to consider the time required for the malaria virus to spread to the host population and vector population.

The remainder of this paper is organized as follows. In Section 2, an improved vector-borne disease model is formulated. In Section 3, the stability of the equilibrium and the existence of local Hopf bifurcation are discussed. In Section 4 , some explicit formulas determining properties bifurcating periodic solutions are obtained by employing the normal form method and the center manifold theorem for the delay differential equations developed by Hassard et al. [20]. Some numerical examples and simulations are performed in Section 5 to demonstrate the main theoretical results, and the conclusions of this paper are summarized in Section 6.

\section{Model Description}

Generally, in the mathematical model of a vector-transmitted disease, the host population size at time $t$, denoted by $N_{1}(t)$, is divided into three subclasses: susceptible, infected, and recovered, with numbers denoted by $S(t), I(t)$, and $R(t)$, respectively. The vector population size at time $t$, given by $N_{2}(t)$, is partitioned into two subclasses: susceptible vectors $M(t)$ and infectious vectors $V(t)$. Based on the models [4$6]$, we consider an improved vector-borne disease model with two delays and reinfection as follows:

$$
\left\{\begin{array}{l}
\frac{d S}{d t}=\Lambda_{1}-b \beta_{1} S\left(t-\tau_{1}\right) V\left(t-\tau_{1}\right)-\mu_{1} S(t) \\
\frac{d I}{d t}=b \beta_{1} S\left(t-\tau_{1}\right) V\left(t-\tau_{1}\right)+\sigma b \beta_{1} R(t) V(t)-\left(\mu_{1}+\gamma\right) I(t) \\
\frac{d R}{d t}=\gamma I(t)-\sigma b \beta_{1} R(t) V(t)-\mu_{1} R(t) \\
\frac{d M}{d t}=\Lambda_{2}-b \beta_{2} M\left(t-\tau_{2}\right) I\left(t-\tau_{2}\right)-\mu_{2} M(t) \\
\frac{d V}{d t}=b \beta_{2} M\left(t-\tau_{2}\right) I\left(t-\tau_{2}\right)-\mu_{2} V(t)
\end{array}\right.
$$

where $\Lambda_{1}$ and $\Lambda_{2}$ are the recruitment rates of the hosts and

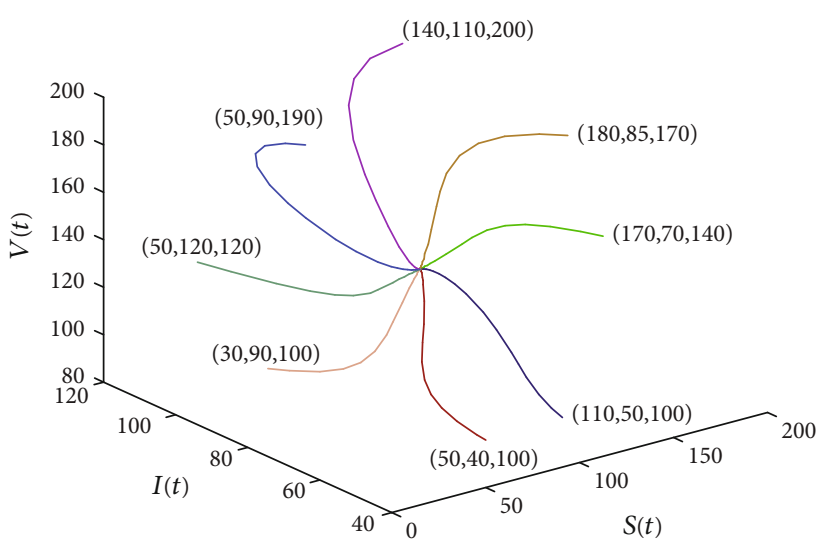

Figure 1: The phase graph of system (2) with $\tau_{1}=\tau_{2}=0$.

vectors, respectively. $b$ is the average number of bites per mosquito per day. $\beta_{1}$ and $\beta_{2}$ represent the infection rates from vector to human and human to vector, respectively. $\mu_{1}$ and $\mu_{2}$ are the natural death rates of the hosts and vectors, respectively. $\sigma(0 \leq \sigma \leq 1)$ is the degree of partial protection of individuals that recovered from primary infection. $\gamma$ is the recovery rate per capita of the infective host population. $\tau_{1}$ and $\tau_{2}$ are two delays, in which $\tau_{1}$ represents the incubation period of the host population, and $\tau_{2}$ denotes the incubation period of the vector population. The term $b \beta_{1} S\left(t-\tau_{1}\right) V(t$ $\left.-\tau_{1}\right)$ represents the incidence number of the susceptible host infections caused by the infective vector at time $t-\tau_{1}$. $b \beta_{2} M\left(t-\tau_{2}\right) I\left(t-\tau_{2}\right)$ denotes the number of infections of the susceptible vectors that bite the infected host at time $t$ $-\tau_{2}$ and become infective at time $t$.

According to [21], system (1) is equivalent to the following model:

$\left\{\begin{array}{l}\frac{d S}{d t}=\Lambda_{1}-b \beta_{1} S\left(t-\tau_{1}\right) V\left(t-\tau_{1}\right)-\mu_{1} S(t), \\ \frac{d I}{d t}=b \beta_{1} S\left(t-\tau_{1}\right) V\left(t-\tau_{1}\right)+\sigma b \beta_{1}\left(\frac{\Lambda_{1}}{\mu_{1}}-S(t)-I(t)\right) V(t)-\left(\mu_{1}+\gamma\right) I(t), \\ \frac{d V}{d t}=b \beta_{2}\left(\frac{\Lambda_{2}}{\mu_{2}}-V\left(t-\tau_{2}\right)\right) I\left(t-\tau_{2}\right)-\mu_{2} V(t) .\end{array}\right.$

Notice that $N_{i}^{\prime}(t)=\Lambda_{i}-\mu_{i} N_{i}(t), i=1,2$, and its solution is $N_{i}(t)=N_{i}(0) e^{-\mu_{i} t}+\left(\Lambda_{i} / \mu_{i}\right)\left(1-e^{-\mu_{i} t}\right)$.

Obviously, $\lim _{t \rightarrow \infty} N_{i}(t)=\Lambda_{i} / \mu_{i}, \quad(i=1,2)$. So, for $t \rightarrow \infty$ and any $\varepsilon>0,0 \leq S+I \leq N_{1}(t)<\left(\Lambda_{1} / \mu_{1}\right)+\varepsilon$ holds by limit theorem, which follows that $0 \leq S+I \leq \Lambda_{1} / \mu_{1}$. Similarly, 0 $\leq V \leq \Lambda_{2} / \mu_{2}$ also holds. Thus, all the solutions of system (2) enter into the region:

$$
\Omega=\left\{(S, I, V) \in R_{+}^{3} \mid 0 \leq S+I \leq \frac{\Lambda_{1}}{\mu_{1}}, 0 \leq V \leq \frac{\Lambda_{2}}{\mu_{2}}, S \geq 0, I \geq 0\right\} .
$$

For the existence of the equilibrium of system (2), according to [5], one can have the following result. 
Lemma 1. For system (2), $E^{0}=\left(\Lambda_{1} / \mu_{1}, 0,0\right)$ is a disease-free equilibrium if $R_{0} \leq 1$, and $E^{*}=\left(S^{*}, I^{*}, V^{*}\right)$ is an endemic equilibrium if $R_{0}>1$, where $R_{0}=\left(b^{2} \Lambda_{1} \Lambda_{2} \beta_{1} \beta_{2}\right) /\left(\mu_{1} \mu_{2}^{2}\left(\mu_{1}\right.\right.$ $+\gamma))$ is the basic reproductive number, $S^{*}=\left(\Lambda_{1} \mu_{2}\left(b \beta_{2} I^{*}+\right.\right.$ $\left.\left.\mu_{2}\right)\right) /\left(b^{2} \Lambda_{2} \beta_{1} \beta_{2} I^{*}+\mu_{1} \mu_{2}\left(b \beta_{2} I^{*}+\mu_{2}\right)\right), \quad V^{*}=\left(b \Lambda_{2} \beta_{2} I^{*}\right) /\left(\mu_{2}\right.$ $\left.\left(b \beta_{2} I^{*}+\mu_{2}\right)\right)$, and $I^{*}$ satisfy the following equation:

$$
P_{2}\left(I^{*}\right)^{2}+P_{1} I^{*}+P_{0}=0
$$

where

$$
\begin{aligned}
P_{2}= & \mu_{1} b^{2} \beta_{2}^{2}\left(b \sigma \beta_{1} \Lambda_{2}+\mu_{1} \mu_{2}\right)\left(b \Lambda_{2} \beta_{1}+\mu_{1} \mu_{2}\right) \\
& +\gamma b^{2} \beta_{2}^{2} \mu_{1} \mu_{2}\left(b \Lambda_{2} \beta_{1}+\mu_{1} \mu_{2}\right), \\
P_{1}= & b \beta_{2} \mu_{1} \mu_{2}^{2}\left(\mu_{1}+\gamma\right)\left(b \Lambda_{2} \beta_{1}+\mu_{1} \mu_{2}\right) \\
& +b \beta_{2} \mu_{1}^{2} \mu_{2}{ }^{2}\left(b \sigma \beta_{1} \Lambda_{2}+\mu_{1} \mu_{2}\right)+b \gamma \beta_{2} \mu_{1}{ }^{2} \mu_{2}{ }^{3} \\
& -b^{3} \Lambda_{1} \Lambda_{2} \beta_{1} \beta_{2}{ }^{2}\left(b \sigma \beta_{1} \Lambda_{2}+\mu_{1} \mu_{2}\right),
\end{aligned}
$$

$$
P_{0}=\mu_{1}^{2} \mu_{2}^{4}\left(\mu_{1}+\gamma\right)\left(1-R_{0}\right)
$$

The disease-free equilibrium $E^{0}$ denotes no infection, and the endemic equilibrium $E^{*}$ represents that the disease will exist and persist. The basic reproductive number $R_{0}$ describes the expected number of secondary infections, which is mainly sensitive to parameters $b$ and $\beta_{1}$ but not affected by parameter $\sigma$ [5]. The process of deriving $R_{0}$ using the nextgeneration method [22-24] is presented in Appendix A.

\section{Stability of Endemic Equilibrium and Hopf Bifurcation}

The Jacobian matrix of the linear system (2) at $E^{*}=\left(S^{*}, I^{*}\right.$, $\left.V^{*}\right)$ is

$$
J=\left(\begin{array}{ccc}
-\mu_{1}-b \beta_{1} V^{*} e^{-\lambda \tau_{1}} & 0 & -b \beta_{1} S^{*} e^{-\lambda \tau_{1}} \\
-\left(\sigma-e^{-\lambda \tau_{1}}\right) b \beta_{1} V^{*} & -\mu_{1}-\gamma-b \sigma \beta_{1} V^{*} & b \beta_{1} S^{*} e^{-\lambda \tau_{1}}+b \sigma \beta_{1}\left(\frac{\Lambda_{1}}{\mu_{1}}-I^{*}-S^{*}\right.
\end{array}\right),
$$

and the characteristic equation $\operatorname{det}(\lambda I-J)=0$ is

$$
\begin{aligned}
\lambda^{3} & +m_{2} \lambda^{2}+m_{1} \lambda+m_{0}+\left(n_{2} \lambda^{2}+n_{1} \lambda+n_{0}\right) e^{-\lambda \tau_{1}} \\
& +\left(q_{2} \lambda^{2}+q_{1} \lambda+q_{0}\right) e^{-\lambda \tau_{2}}+\left(r_{1} \lambda+r_{0}\right) e^{-\lambda\left(\tau_{1}+\tau_{2}\right)}=0
\end{aligned}
$$

where

$$
\begin{gathered}
m_{2}=\mu_{2}+2 \mu_{1}+\gamma+b \sigma \beta_{1} V^{*}, \\
m_{1}=\left(\mu_{1}+\gamma+b \sigma \beta_{1} V^{*}\right)\left(\mu_{1}+\mu_{2}\right)+\mu_{1} \mu_{2}, \\
m_{0}=\mu_{1} \mu_{2}\left(\mu_{1}+\gamma+b \sigma \beta_{1} V^{*}\right), \\
n_{2}=b \beta_{1} V^{*} \\
n_{1}=b \beta_{1} V^{*}\left(\mu_{2}+\mu_{1}+\gamma+b \sigma \beta_{1} V^{*}\right), \\
n_{0}=b \beta_{1} V^{*}\left(\mu_{1}+\gamma+b \sigma \beta_{1} V^{*}\right) \mu_{2}, \\
q_{2}=b \beta_{2} I^{*}
\end{gathered}
$$

$$
\begin{aligned}
q_{1}= & b \beta_{2} I^{*}\left(2 \mu_{1}+\gamma+b \sigma \beta_{1} V^{*}\right)-b \sigma \beta_{1}\left(\frac{\Lambda_{1}}{\mu_{1}}-I^{*}-S^{*}\right) b \beta_{2} \\
& \cdot\left(\frac{\Lambda_{2}}{\mu_{2}}-V^{*}\right),
\end{aligned}
$$$$
q_{0}=b \beta_{2} I^{*} \mu_{1}\left(\mu_{1}+\gamma+b \sigma \beta_{1} V^{*}\right)-b \sigma \beta_{1}\left(\frac{\Lambda_{1}}{\mu_{1}}-I^{*}-S^{*}\right) b \beta_{2}
$$$$
\cdot\left(\frac{\Lambda_{2}}{\mu_{2}}-V^{*}\right) \mu_{1}
$$$$
r_{1}=b^{2} \beta_{1} \beta_{2} V^{*} I^{*}-b \beta_{2}\left(\frac{\Lambda_{2}}{\mu_{2}}-V^{*}\right) b \beta_{1} S^{*},
$$$$
\begin{aligned}
r_{0}= & b^{2} \beta_{1} \beta_{2} V^{*} I^{*}\left(\mu_{1}+\gamma+b \sigma \beta_{1} V^{*}\right)-b^{3} \beta_{1}^{2} \beta_{2} S^{*} \sigma V^{*}\left(\frac{\Lambda_{2}}{\mu_{2}}-V^{*}\right) \\
& -\left[b^{3} \beta_{1}^{2} \beta_{2} \sigma V^{*}\left(\frac{\Lambda_{1}}{\mu_{1}}-I^{*}-S^{*}\right)\left(\frac{\Lambda_{2}}{\mu_{2}}-V^{*}\right)\right. \\
& \left.+\mu_{1} b^{2} \beta_{1} \beta_{2} S^{*}\left(\frac{\Lambda_{2}}{\mu_{2}}-V^{*}\right)\right] .
\end{aligned}
$$

Case (1). $\tau_{1}=\tau_{2}=0$. 

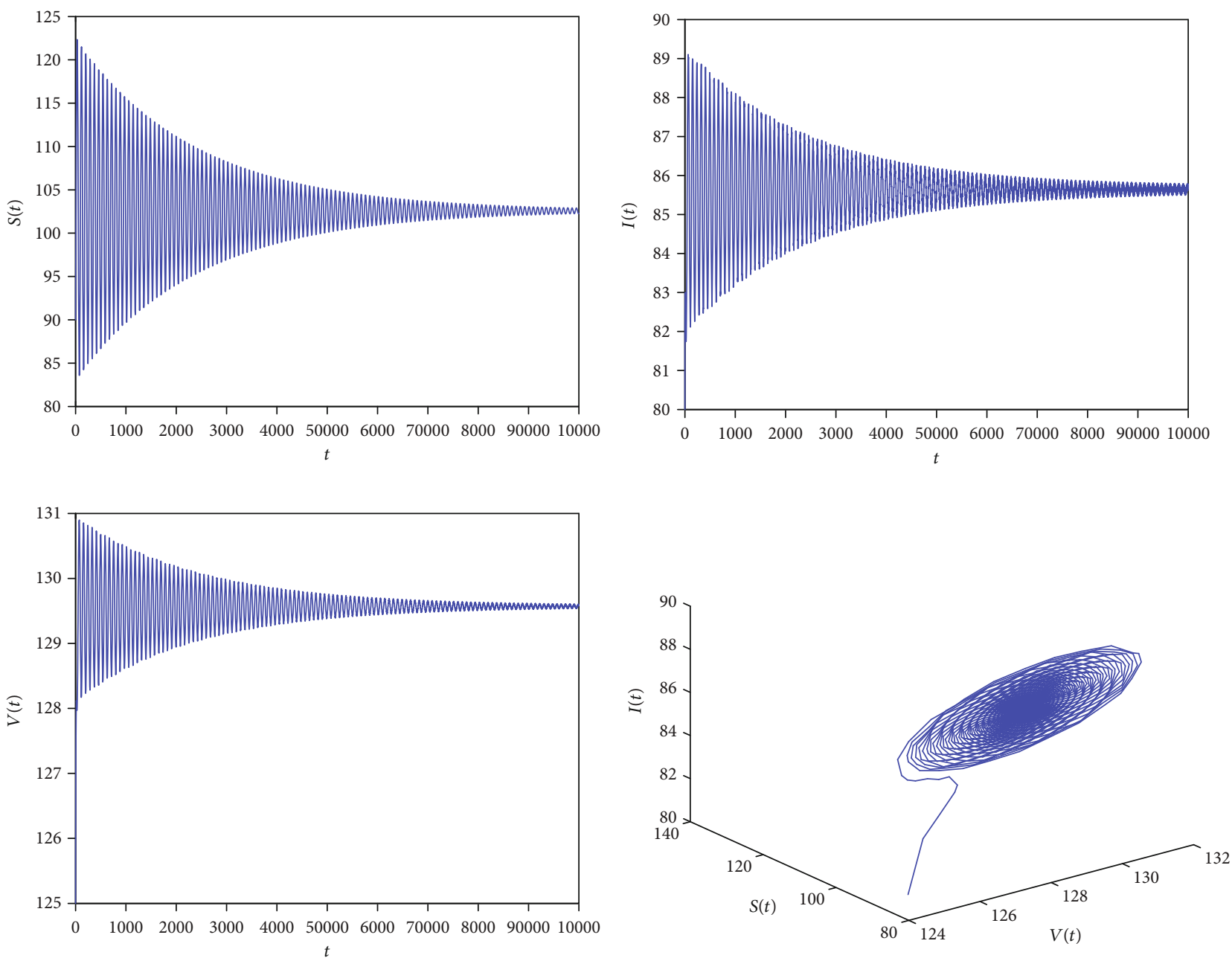

FIgUre 2: The endemic equilibrium $E^{*}$ is asymptotically stable with $\tau_{1}=23<\tau_{10}$.

Characteristic equation (7) becomes

$$
\lambda^{3}+A_{12} \lambda^{2}+A_{11} \lambda+A_{10}=0
$$

where $\mathrm{A}_{12}=m_{2}+n_{2}+q_{2}, \mathrm{~A}_{11}=m_{1}+n_{1}+q_{1}+r_{1}$, and $\mathrm{A}_{10}$ $=m_{0}+n_{0}+q_{0}+r_{0}$.

When the following condition (H1) holds, all roots of equation (9) have negative real parts.

$$
(\mathrm{H} 1): A_{12}>0, A_{11}>0, A_{10}>0, A_{12} A_{11}-A_{10}>0 \text {. }
$$

Hence, according to the Routh-Hurwitz criterion, the following conclusion can be drawn.

Theorem 2. If (H1) holds, the endemic equilibrium $E^{*}$ is locally asymptotically stable when $\tau_{1}=\tau_{2}=0$.

Case (2). $\tau_{1}>0, \tau_{2}=0$.
The characteristic equation (7) becomes

$$
\lambda^{3}+A_{22} \lambda^{2}+A_{21} \lambda+A_{20}+\left(B_{22} \lambda^{2}+B_{21} \lambda+B_{20}\right) e^{-\lambda \tau_{1}}=0
$$

where $\mathrm{A}_{22}=m_{2}+q_{2}, \mathrm{~A}_{21}=m_{1}+q_{1}, \mathrm{~A}_{20}=m_{0}+q_{0}, B_{22}=n_{2}$, $B_{21}=n_{1}+r_{1}$, and $B_{20}=n_{0}+r_{0}$.

Suppose $\lambda=i \omega_{1}\left(\omega_{1}>0\right)$ is a root of equation (11), replacing it into equation (11) and separating the real and imaginary parts, then, we can obtain

$$
\left\{\begin{array}{l}
A_{22} \omega_{1}^{2}-A_{20}=\left(B_{20}-B_{22} \omega_{1}^{2}\right) \cos \omega_{1} \tau_{1}+B_{21} \omega_{1} \sin \omega_{1} \tau_{1}, \\
\omega_{1}^{3}-A_{21} \omega_{1}=-\left(B_{20}-B_{22} \omega_{1}^{2}\right) \sin \omega_{1} \tau_{1}+B_{21} \omega_{1} \cos \omega_{1} \tau_{1} .
\end{array}\right.
$$

It follows that

$$
\begin{aligned}
& \omega_{1}^{6}+\left(A_{22}^{2}-2 A_{21}-B_{22}{ }^{2}\right) \omega_{1}^{4}+\left(A_{21}^{2}-B_{21}^{2}-2 A_{20} A_{22}+2 B_{20} B_{22}\right) \omega_{1}^{2} \\
& +A_{20}{ }^{2}-B_{20}{ }^{2}=0 .
\end{aligned}
$$



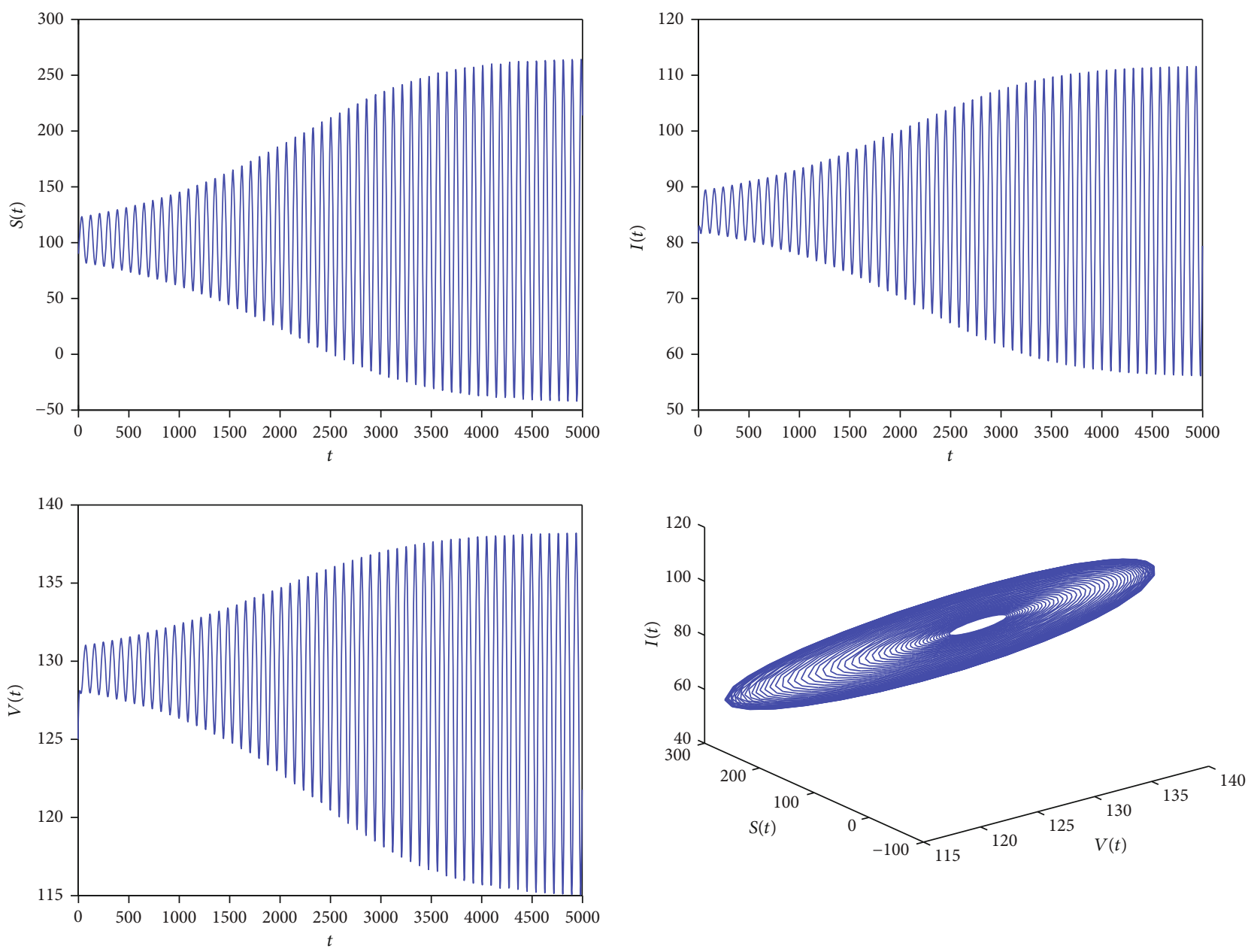

Figure 3: The endemic equilibrium $E^{*}$ is unstable with $\tau_{1}=24>\tau_{10}$.

Let $z=\omega_{1}{ }^{2}, p_{2}=A_{22}{ }^{2}-2 A_{21}-B_{22}{ }^{2}, p_{1}=A_{21}{ }^{2}-B_{21}{ }^{2}-2$ $A_{20} A_{22}+2 B_{20} B_{22}$, and $p_{0}=A_{20}{ }^{2}-B_{20}{ }^{2}$, then, equation (13) is equivalent to

$$
f(z)=z^{3}+p_{2} z^{2}+p_{1} z+p_{0}=0 .
$$

It is easy to get that the two roots of $f^{\prime}(z)=3 z^{2}+2 p_{2} z$ $+p_{1}=0$ are $z_{1}=(1 / 3)\left(-p_{2}+\sqrt{p_{2}^{2}-3 p_{1}}\right)$ and $z_{2}=(1 / 3)\left(-p_{2}\right.$ $\left.-\sqrt{p_{2}^{2}-3 p_{1}}\right)$.

According to $[25,26]$, we can get the conditions that equation (14) exists positive roots.

Lemma 3. For equation (14),

(i) If $p_{0}<0$, then equation (14) has at least one positive root

(ii) If $p_{0} \geq 0$ and $\Delta_{1}=p_{2}^{2}-3 p_{1} \leq 0$, then equation (14) has no positive roots

(iii) If $p_{0} \geq 0$ and $\Delta_{1}=p_{2}^{2}-3 p_{1}>0$, then equation (14) has positive roots if and only if $z_{1}=(1 / 3)\left(-p_{2}+\right.$ $\left.\sqrt{\Delta_{1}}\right)>0$ and $f\left(z_{1}\right) \leq 0$
By Lemma 3, it is easy to see that the stability of $E^{*}$ will not change when $\tau$ changes if (ii) is set up. However, the stability may change when $\tau$ crosses through some critical values if equation (14) has a positive root.

Assume that $z_{1}, z_{2}$, and $z_{3}$ are three positive roots of equation (14), then, there are three positive numbers $\omega_{11}=$ $\sqrt{z_{1}}, \omega_{12}=\sqrt{z_{2}}$, and $\omega_{13}=\sqrt{z_{3}}$. It follows from equation (12) that

$\cos \omega_{1} \tau_{1}=\frac{\left(A_{22} \omega_{1}{ }^{2}-A_{20}\right)\left(B_{20}-B_{22} \omega_{1}^{2}\right)+B_{21} \omega_{1}\left(\omega_{1}^{3}-A_{21} \omega_{1}\right)}{\left(B_{20}-B_{22} \omega_{1}^{2}\right)^{2}+B_{21}{ }^{2} \omega_{1}{ }^{2}}$.

Denote

$\tau_{1 k}^{(j)}=\frac{1}{\omega_{1 k}} \arccos \left\{\frac{\left(A_{22} \omega_{1}{ }^{2}-A_{20}\right)\left(B_{20}-B_{22} \omega_{1}{ }^{2}\right)+B_{21} \omega_{1}\left(\omega_{1}{ }^{3}-A_{21} \omega_{1}\right)}{\left(B_{20}-B_{22} \omega_{1}^{2}\right)^{2}+B_{21}{ }^{2} \omega_{1}{ }^{2}}\right\}+\frac{2 j \pi}{\omega_{1 k}}$,

where $j=0,1,2, \cdots, k=1,2,3$, then, $\pm i \omega_{1 k}$ is a pair of purely imaginary roots of equation (11) for $\tau_{1}=\tau_{1 k}^{(j)}$. Let $\tau_{10}=$ 

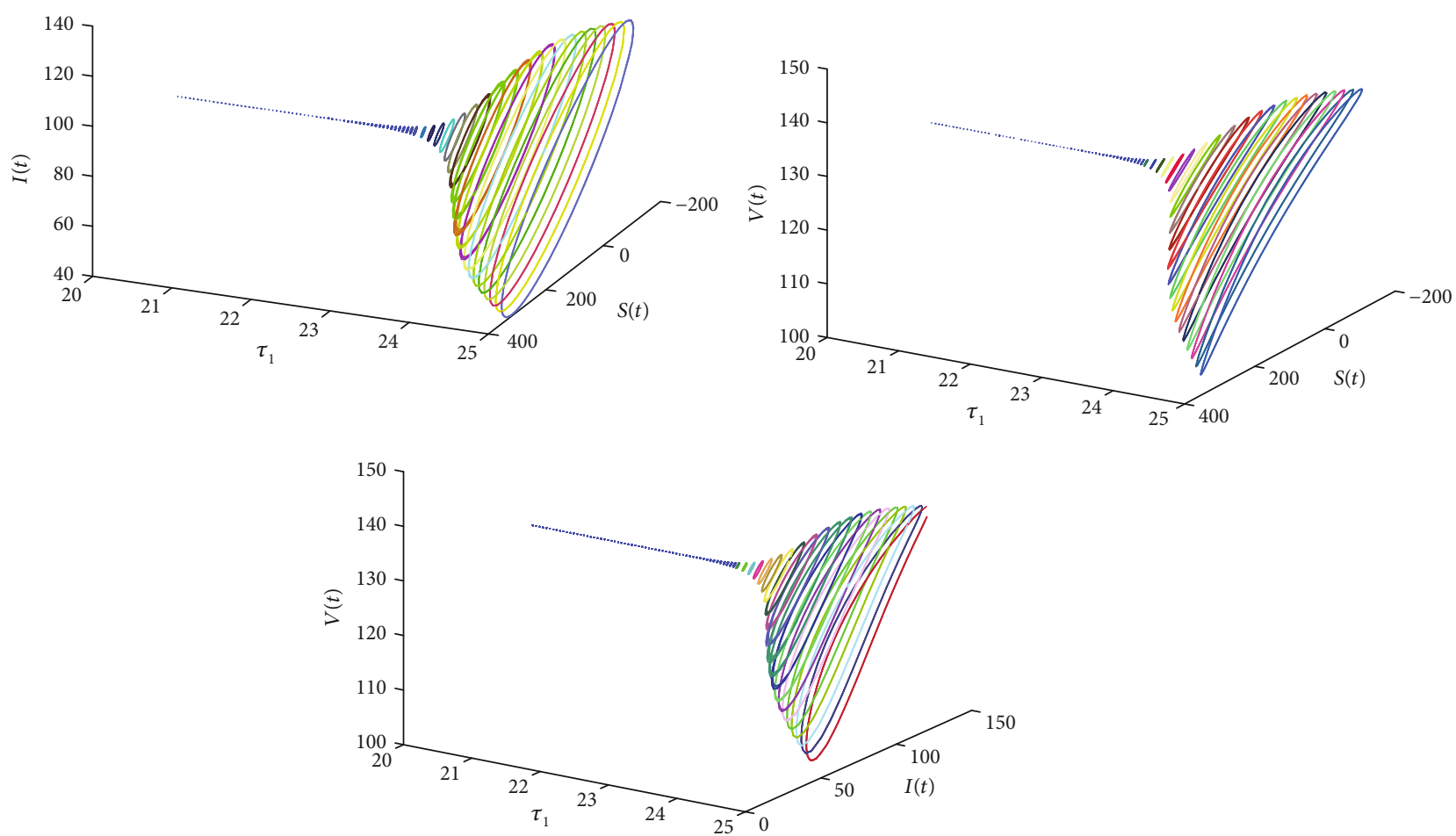

FIGURE 4: The bifurcation diagrams with $\tau_{1}$ as parameter and $\tau_{2}=0$. A Hopf bifurcation exists at $\tau_{1}=\tau_{10}$.

$\min _{k \in\{1,2,3\}}\left\{\tau_{1 k}^{(0)}\right\}, \omega_{10}$ be the $\omega_{1 k}$ that corresponds to the minimum $\tau_{1 k}^{(0)}$, and $\lambda\left(\tau_{1}\right)=\alpha\left(\tau_{1}\right)+i \omega\left(\tau_{1}\right)$ be the root of equation (11) near $\tau_{1}=\tau_{1 k}^{(j)}$ satisfying $\alpha\left(\tau_{1 k}^{(j)}\right)=0$ and $\omega\left(\tau_{1 k}^{(j)}\right)=\omega_{1 k}$.

Furthermore, we consider the transversality condition. By differentiating both sides of equation (11) with respect to $\tau_{1}$, we get

$$
\left[\frac{d \lambda}{d \tau_{1}}\right]^{-1}=\frac{3 \lambda^{2}+2 A_{22} \lambda+A_{21}}{-\lambda\left(\lambda^{3}+A_{22} \lambda^{2}+A_{21} \lambda+A_{20}\right)}+\frac{2 B_{22} \lambda+B_{21}}{\lambda\left(B_{22} \lambda^{2}+B_{21} \lambda+B_{20}\right)}-\frac{\tau_{1}}{\lambda} .
$$

It follows that

$$
\begin{aligned}
\operatorname{Re} & \left\{\left[\frac{d \lambda}{d \tau_{1}}\right]_{\tau_{1}=\tau_{10}}^{-1}\right\}=\operatorname{Re}\left\{\frac{\left(3 \lambda^{2}+2 A_{22} \lambda+A_{21}\right) \mathrm{e}^{\lambda \tau_{1}}+2 B_{22} \lambda+B_{21}}{\lambda\left(B_{22} \lambda^{2}+B_{21} \lambda+B_{20}\right)}-\frac{\tau_{1}}{\lambda}\right\}_{\tau_{1}=\tau_{10}} \\
& =\frac{\left[3 \omega_{1}^{4}+\left(2 A_{22}^{2}-4 A_{21}-2 B_{22}^{2}\right) \omega_{1}^{2}+A_{21}^{2}-2 A_{20} A_{22}-B_{21}^{2}+2 B_{20} B_{22}\right] \omega_{1}^{2}}{B_{21}^{2} \omega_{1}^{4}+\left(B_{20}-B_{22} \omega_{1}^{2}\right)^{2} \omega_{1}^{2}} \\
& =\frac{\omega_{1}^{2} f^{\prime}\left(\omega_{1}^{2}\right)}{B_{21}^{2} \omega_{1}^{4}+\left(B_{20}-B_{22} \omega_{1}^{2}\right)^{2} \omega_{1}^{2}} .
\end{aligned}
$$

Thus,

$\operatorname{sign}\left\{\left.\frac{d(\operatorname{Re} \lambda)}{d \tau_{1}}\right|_{\tau_{1}=\tau_{10}}\right\}=\operatorname{sign}\left\{\operatorname{Re}\left\{\left[\frac{d \lambda}{d \tau_{1}}\right]_{\tau_{1}=\tau_{10}}^{-1}\right\}\right\}=\operatorname{sign}\left\{f^{\prime}\left(\omega_{1}^{2}\right)\right\}$.

The transversality condition is satisfied when $f^{\prime}\left(\omega_{1}^{2}\right) \neq 0$.
According to [27], and applying Lemma 3 and the above transversal condition to system (2), the following theorem is obtained.

Theorem 4. For system (2), when $\tau_{2}=0$,

(i) if $p_{0} \geq 0$ and $\Delta_{1}=p_{2}^{2}-3 p_{1} \leq 0$ hold, then, the endemic equilibrium $E^{*}$ is locally asymptotically stable for all $\tau_{1}>0$

(ii) if $p_{0}<0$ or $p_{0} \geq 0, \Delta_{1}=p_{2}^{2}-3 p_{1}>0, z_{1}>0$, and $f\left(z_{1}\right.$ )$\leq 0$, then, the endemic equilibrium $E^{*}$ is locally asymptotically stable for $\tau_{1} \in\left(0, \tau_{10}\right)$

(iii) if the conditions of (ii) and $f^{\prime}\left(z_{1}\right) \neq 0$ hold, then, it undergoes a Hopf bifurcation at $E^{*}$ when $\tau_{1}=\tau_{10}$

Case (3). $\tau_{1}>0, \tau_{2}>0$.

Fixing $\tau_{1}$ in its stable interval and regarding $\tau_{2}$ as a parameter, we analyze the roots of characteristic equation (7). Let $\lambda=i \omega(\omega>0)$ be a characteristic root of equation (7). By performing some calculations as those in case (2), we can obtain

$$
\begin{aligned}
& \left(\lambda^{3}+m_{2} \lambda^{2}+m_{1} \lambda+m_{0}\right) e^{\lambda \tau_{2}}+\left(n_{2} \lambda^{2}+n_{1} \lambda+n_{0}\right) e^{-\lambda \tau_{1}} e^{\lambda \tau_{2}} \\
& +\left(q_{2} \lambda^{2}+q_{1} \lambda+q_{0}\right)+\left(r_{1} \lambda+r_{0}\right) e^{-\lambda \tau_{1}}=0
\end{aligned}
$$



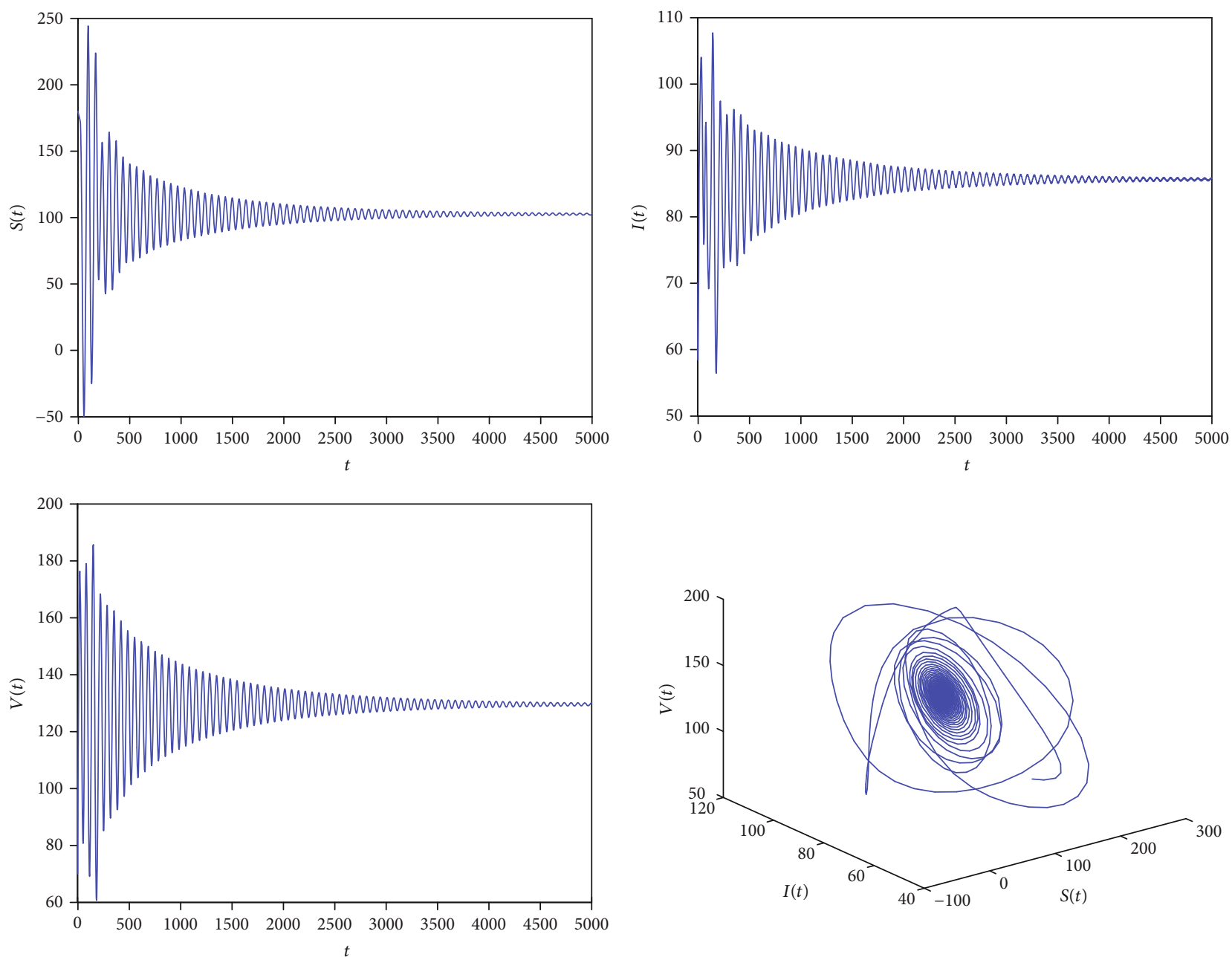

FIgURE 5: The endemic equilibrium $E^{*}$ is asymptotically stable with $\tau_{1}=23, \tau_{2}=21<\tau_{2}^{*}$.

$$
\begin{gathered}
\left\{\begin{array}{l}
A_{31} \cos \omega \tau_{2}+A_{32} \sin \omega \tau_{2}=A_{34}, \\
A_{31} \sin \omega \tau_{2}-A_{32} \cos \omega \tau_{2}=A_{35},
\end{array}\right. \\
\omega^{6}+k_{5} \omega^{5}+k_{4} \omega^{4}+k_{3} \omega^{3}+k_{2} \omega^{2}+k_{1} \omega+k_{0}=0,
\end{gathered}
$$

where

$$
\begin{gathered}
A_{31}=\left(n_{0}-n_{2} \omega^{2}\right) \cos \omega \tau_{1}+n_{1} \omega \sin \omega \tau_{1}+m_{0}-m_{2} \omega^{2}, \\
A_{32}=\left(n_{0}-n_{2} \omega^{2}\right) \sin \omega \tau_{1}-n_{1} \omega \cos \omega \tau_{1}+\omega^{3}-m_{1} \omega, \\
A_{34}=-r_{1} \omega \sin \omega \tau_{1}-r_{0} \cos \omega \tau_{1}+q_{2} \omega^{2}-q_{0}, \\
A_{35}=-r_{1} \omega \cos \omega \tau_{1}+r_{0} \sin \omega \tau_{1}-q_{1} \omega, \\
k_{0}=n_{0}^{2}+m_{0}^{2}-r_{0}^{2}-q_{0}^{2}+2 n_{0} m_{0} \cos \omega \tau_{1}-2 r_{0} q_{0} \cos \omega \tau_{1}, \\
k_{1}=2\left(n_{1} m_{0}-n_{0} m_{1}-r_{1} q_{0}+r_{0} q_{1}\right) \sin \omega \tau_{1},
\end{gathered}
$$

$$
\begin{gathered}
k_{2}=n_{1}^{2}+m_{1}^{2}-2 n_{0} n_{2}-2 n_{0} m_{2} \cos \omega \tau_{1}-2 n_{2} m_{0} \cos \omega \tau_{1} \\
-2 m_{0} m_{2}+2 n_{1} m_{1} \cos \omega \tau_{1}-r_{1}^{2}+2 r_{0} q_{2} \cos \omega \tau_{1}+2 q_{0} q_{2} \\
-2 r_{1} q_{1} \cos \omega \tau_{1}-q_{1}^{2}, \\
k_{3}=2\left(n_{0}+m_{1} n_{2}-n_{1} m_{2}+r_{1} q_{2}\right) \sin \omega \tau_{1}, \\
k_{4}=n_{2}^{2}+m_{2}^{2}+2 n_{2} m_{2} \cos \omega \tau_{1}-2 n_{1} \cos \omega \tau_{1}-2 m_{1}-q_{2}^{2}, \\
k_{5}=-2 n_{2} \sin \omega \tau_{1} .
\end{gathered}
$$

Furthermore, we suppose that

$(\mathrm{H} 2)$ : equation (22) has finite positive roots $\omega_{2}^{(i)},(i=1,2$ $, \cdots, k)$.

Then, for every fixed $\omega_{2}^{(i)}$, there exists a sequence of critical values $\tau_{2 i}^{(j)}$ such that equation (22) holds, where

$$
\tau_{2 i}^{(j)}=\frac{1}{\omega_{2}^{(i)}} \arccos \left\{\frac{A_{31} A_{34}-A_{32} A_{35}}{A_{31}^{2}+A_{32}^{2}}\right\}+\frac{2 j \pi}{\omega_{2}^{(i)}}, \quad\left(i=1,2, \cdots, k ; j \in \mathbb{N}_{0}\right) .
$$



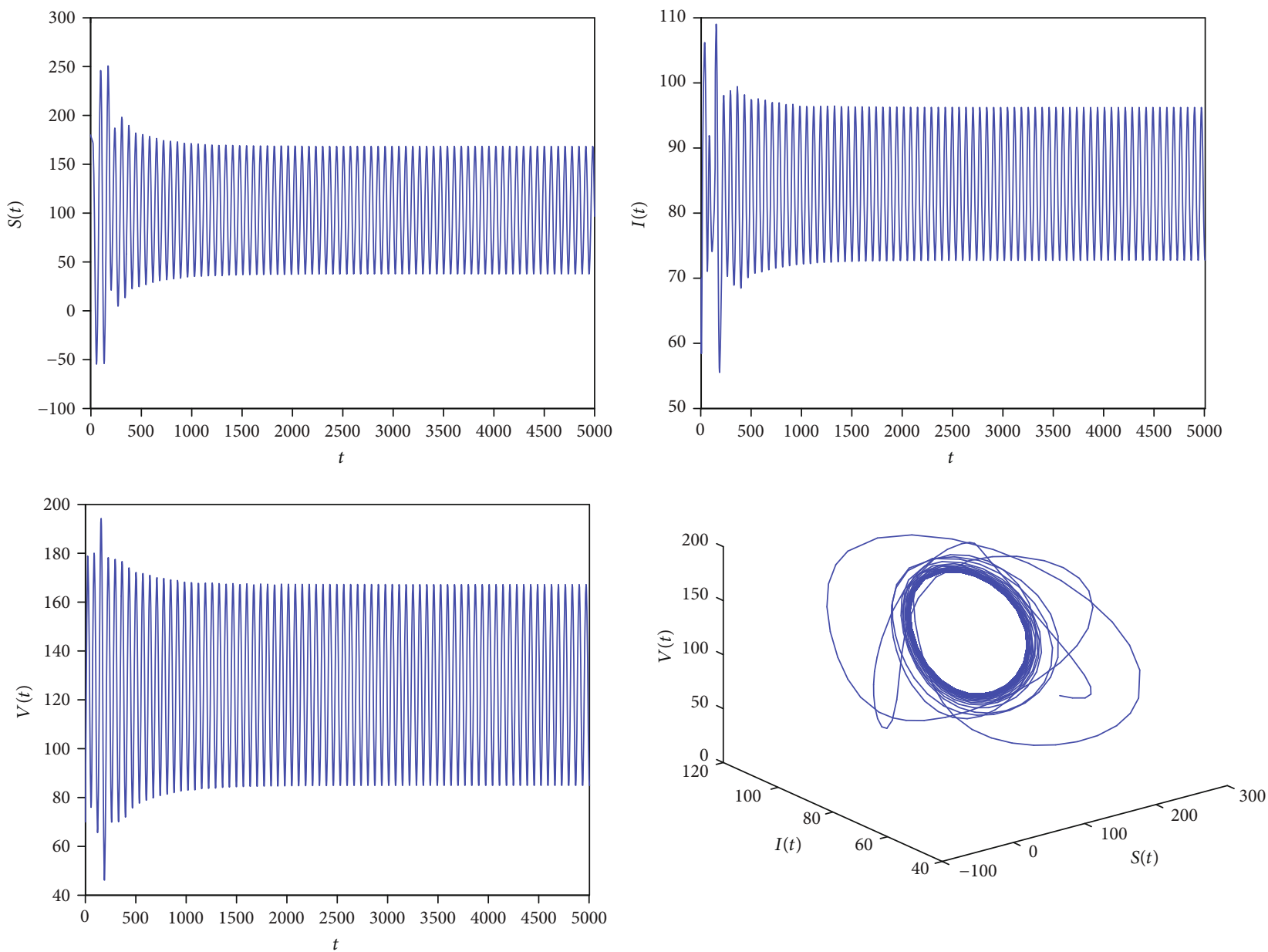

FIgURE 6: The endemic equilibrium $E^{*}$ is unstable with $\tau_{1}=23$ and $\tau_{2}=22>\tau_{2}^{*}$.

Let $\tau_{2}^{*}=\min \left\{\tau_{2 i}^{0} \mid i=1,2, \cdots, k\right\}$, when $\tau_{2}=\tau_{2}^{*}$, then equation (7) has a pair of purely imaginary roots $\pm i \omega_{2}^{*}$.

Next, we also check the transversality condition. By taking the differentiation of (20) with respect to $\tau_{2}$ and further calculating, we can obtain

$$
\left[\frac{d \lambda}{d \tau_{2}}\right]^{-1}=-\frac{f_{31}(\lambda)}{f_{32}(\lambda)}+\frac{\tau_{2}}{\lambda},
$$

where

$$
\begin{aligned}
f_{31}(\lambda)= & \left(3 \lambda^{2}+2 m_{2} \lambda+m_{1}\right) e^{\lambda \tau_{2}}+\left(2 n_{2} \lambda+n_{1}\right) e^{\lambda\left(\tau_{2}-\tau_{1}\right)} \\
& -\left(n_{2} \lambda^{2}+n_{1} \lambda+n_{0}\right) \tau_{1} e^{\lambda\left(\tau_{2}-\tau_{1}\right)}+\left(2 q_{2} \lambda+q_{1}\right) \\
& +r_{1} e^{-\lambda \tau_{1}}-\left(r_{1} \lambda+r_{0}\right) \tau_{1} e^{-\lambda \tau_{1}}
\end{aligned}
$$

where

$$
\begin{aligned}
Q_{31}= & {\left[\left(n_{0} \omega_{2}^{*}-n_{2}\left(\omega_{2}^{*}\right)^{3}\right) \sin \omega_{2}^{*} \tau_{1}-n_{1}\left(\omega_{2}^{*}\right)^{2} \cos \omega_{2}^{*} \tau_{1}+\left(\omega_{2}^{*}\right)^{4}\right.} \\
& \left.-m_{1}\left(\omega_{2}^{*}\right)^{2}\right] \cos \omega_{2}^{*} \tau_{2}^{*}-\left[\left(n_{0} \omega_{2}^{*}-n_{2}\left(\omega_{2}^{*}\right)^{3}\right) \cos \omega_{2}^{*} \tau_{1}\right. \\
& \left.+n_{1}\left(\omega_{2}^{*}\right)^{2} \sin \omega_{2}^{*} \tau_{1}+m_{0} \omega_{2}^{*}-m_{2}\left(\omega_{2}^{*}\right)^{3}\right] \sin \omega_{2}^{*} \tau_{2}^{*}
\end{aligned}
$$

$$
\begin{aligned}
Q_{32}= & {\left[\left(n_{0} \omega_{2}^{*}-n_{2}\left(\omega_{2}^{*}\right)^{3}\right) \sin \omega_{2}^{*} \tau_{1}-n_{1}\left(\omega_{2}^{*}\right)^{2} \cos \omega_{2}^{*} \tau_{1}+\left(\omega_{2}^{*}\right)^{4}\right.} \\
& \left.-m_{1}\left(\omega_{2}^{*}\right)^{2}\right] \sin \omega_{2}^{*} \tau_{2}^{*}+\left[\left(n_{0} \omega_{2}^{*}-n_{2}\left(\omega_{2}^{*}\right)^{3}\right) \cos \omega_{2}^{*} \tau_{1}\right. \\
& \left.+n_{1}\left(\omega_{2}^{*}\right)^{2} \sin \omega_{2}^{*} \tau_{1}-m_{2}\left(\omega_{2}^{*}\right)^{3}+m_{0} \omega_{2}^{*}\right] \cos \omega_{2}^{*} \tau_{2}^{*}
\end{aligned}
$$

$f_{32}(\lambda)=\left(\lambda^{4}+m_{2} \lambda^{3}+m_{1} \lambda^{2}+m_{0} \lambda\right) e^{\lambda \tau_{2}}+\left(n_{2} \lambda^{3}+n_{1} \lambda^{2}+n_{0} \lambda\right) e^{\lambda\left(\tau_{2}-\tau_{1}\right)}$

Thus, we can get

$$
\operatorname{Re}\left\{\left[\frac{d \lambda}{d \tau_{2}}\right]_{\tau_{2}=\tau_{2}^{*}}^{-1}\right\}=-\frac{P_{31} Q_{31}+P_{32} Q_{32}}{Q_{31}^{2}+Q_{32}^{2}},
$$




$$
\begin{aligned}
P_{32}= & {\left[\left(\tau_{1} n_{2}\left(\omega_{2}^{*}\right)^{2}-\tau_{1} n_{0}+n_{1}\right) \cos \omega_{2}^{*} \tau_{1}\right.} \\
& \left.+\left(2 n_{2}-\tau_{1} n_{1}\right) \omega_{2}^{*} \sin \omega_{2}^{*} \tau_{1}-3\left(\omega_{2}^{*}\right)^{2}+m_{1}\right] \sin \omega_{2}^{*} \tau_{2}^{*} \\
& -\left[\left(\tau_{1} n_{2}\left(\omega_{2}^{*}\right)^{2}-\tau_{1} n_{0}+n_{1}\right) \sin \omega_{2}^{*} \tau_{1}\right. \\
& \left.-\left(2 n_{2}-\tau_{1} n_{1}\right) \omega_{2}^{*} \cos \omega_{2}^{*} \tau_{1}-2 m_{2} \omega_{2}^{*}\right] \cos \omega_{2}^{*} \tau_{2}^{*} \\
& -\left(r_{1}-r_{0} \tau_{1}\right) \sin \omega_{2}^{*} \tau_{1}-\tau_{1} r_{1} \omega_{2}^{*} \cos \omega_{2}^{*} \tau_{1}+2 q_{2} \omega_{2}^{*}
\end{aligned}
$$

Therefore, when

(H3): $P_{31} Q_{31}+P_{32} Q_{32} \neq 0$ holds, then $\operatorname{Re}\left\{\left[d \lambda / d \tau_{2}\right]_{\tau_{2}=\tau_{2}^{*}}^{-1}\right.$ \}$\neq 0$, i.e., the transversality condition is satisfied.

According to the above discussions and based on [27], we can get the following result.

Theorem 5. Let $\tau_{1} \in\left(0, \tau_{10}\right)$, if (H2) and (H3) hold, then, the endemic equilibrium $E^{*}$ is locally asymptotically stable for $\tau_{2}$ $\in\left[0, \tau_{2}^{*}\right)$ and is unstable for $\tau_{2} \in\left(\tau_{2}^{*},+\infty\right)$. System (2) undergoes Hopf bifurcation at the endemic equilibrium $E^{*}$ for $\tau_{2}=\tau_{2}^{*}$.

\section{Properties of Hopf Bifurcation}

From the analysis in the last section, we can see that for some critical values of delays, system (2) can occur in a series of periodic solutions at the equilibrium. On the basis of Theorem 5, we will employ the normal form method and the center manifold theorem introduced by Hassard et al. [20] to provide the properties of bifurcating periodic solutions in this section. Without loss of generality, we assume that $\tau_{1}^{*}$ $<\tau_{2}^{*}, \tau_{1}^{*} \in\left(0, \tau_{10}\right), \tau_{2}=\tau_{2}^{*}+\mu, u_{1}(t)=S(\tau t)-S^{*}, u_{2}(t)=I($ $\tau t)-I^{*}$, and $u_{3}(t)=V(\tau t)-V^{*}$. Then, equation (2) can be rewritten into the following functional differential equation in the Banach Space $C=C\left([-1,0], \mathbb{R}^{3}\right)$.

$$
\dot{u}(t)=L_{\mu}\left(u_{t}\right)+F\left(\mu, u_{t}\right)
$$

where $u(t)=\left(u_{1}(t), u_{2}(t), u_{3}(t)\right)^{T} \in \mathbb{R}^{3}, u_{t}(\theta)=u(t+\theta), L_{\mu}$ $: C \rightarrow \mathbb{R}^{3}$, and $F(\mu, \cdot): \mathbb{R} \times C \rightarrow \mathbb{R}^{3}$ are defined as follows:

$$
\begin{gathered}
L_{\mu}(\phi)=\left(\tau_{2}^{*}+\mu\right)\left[A^{\prime} \phi(0)+B^{\prime} \phi\left(-\frac{\tau_{1}^{*}}{\tau_{2}^{*}}\right)+C^{\prime} \phi(-1)\right], \\
F(\mu, \phi)=\left(\tau_{2}^{*}+\mu\right)\left(F_{1}, F_{2}, F_{3}\right)^{T},
\end{gathered}
$$

$$
\begin{gathered}
\phi(\theta)=\left(\phi_{1}(\theta), \phi_{2}(\theta), \phi_{3}(\theta)\right)^{T} \in C, \\
A^{\prime}=\left(\begin{array}{ccc}
-\mu_{1} & 0 \\
-\sigma b \beta_{1} V^{*} & -\left(\mu_{1}+\gamma+b \sigma \beta_{1} V^{*}\right) & b \sigma \beta_{1}\left(\frac{\Lambda_{1}}{\mu_{1}}-I^{*}-S^{*}\right) \\
0 & -\mu_{2}
\end{array}\right), \\
B^{\prime}=\left(\begin{array}{ccc}
-b \beta_{1} V^{*} & 0 & -b \beta_{1} S^{*} \\
b \beta_{1} V^{*} & 0 & b \beta_{1} S^{*} \\
0 & 0 & 0
\end{array}\right), \\
C^{\prime}=\left(\begin{array}{ccc}
0 & 0 & 0 \\
0 & 0 & 0 \\
0 & b \beta_{2}\left(\frac{\Lambda_{2}}{\mu_{2}}-V^{*}\right.
\end{array}\right) \\
F_{2}=b \beta_{1} \phi_{1}\left(-\frac{\tau_{1}^{*}}{\tau_{2}^{*}}\right) \beta_{3} I^{*}\left(-\frac{\tau_{1}^{*}}{\tau_{2}^{*}}\right)-b \sigma \beta_{1} \phi_{1}(0) \phi_{3}(0)-b \sigma \beta_{1} \phi_{2}(0) \phi_{3}(0), \\
F_{3}=-b \beta_{2} \phi_{2}(-1) \phi_{3}(-1) .
\end{gathered}
$$

Based on the Riesz representation theorem, we know that there exists a function of bounded variation components $\eta($ $\theta, \mu), \theta \in[-1,0]$, such that

$$
L_{\mu}(\phi)=\int_{-1}^{0} \mathrm{~d} \eta(\theta, \mu) \phi(\theta), \phi(\theta) \in C\left([-1,0], \mathbb{R}^{3}\right) .
$$

In fact, we can take

$$
\eta(\theta, \mu)= \begin{cases}\left(\tau_{2}^{*}+\mu\right)\left(A^{\prime}+B^{\prime}+C^{\prime}\right), & \theta=0, \\ \left(\tau_{2}^{*}+\mu\right)\left(B^{\prime}+C^{\prime}\right), & \theta \in\left[-\frac{\tau_{1}^{*}}{\tau_{2}^{*}}, 0\right], \\ \left(\tau_{2}^{*}+\mu\right) C^{\prime}, & \theta \in\left(-1,-\frac{\tau_{1}^{*}}{\tau_{2}^{*}}\right), \\ 0, & \theta=-1 .\end{cases}
$$

Define $A(\mu)$ and $R(\mu)$ by

$$
\begin{aligned}
& A(\mu) \phi= \begin{cases}\frac{\mathrm{d} \phi(\theta)}{d \theta}, & \theta \in[-1,0), \\
\int_{-1}^{0} \mathrm{~d} \eta(s, \mu) \phi(s), & \theta=0,\end{cases} \\
& R(\mu) \phi= \begin{cases}0, & \theta \in[-1,0), \\
F(\mu, \phi), & \theta=0 .\end{cases}
\end{aligned}
$$

Then, system (29) can be further represented as

$$
\dot{u}(t)=A(\mu) u_{t}+R(\mu) u_{t}
$$

where 

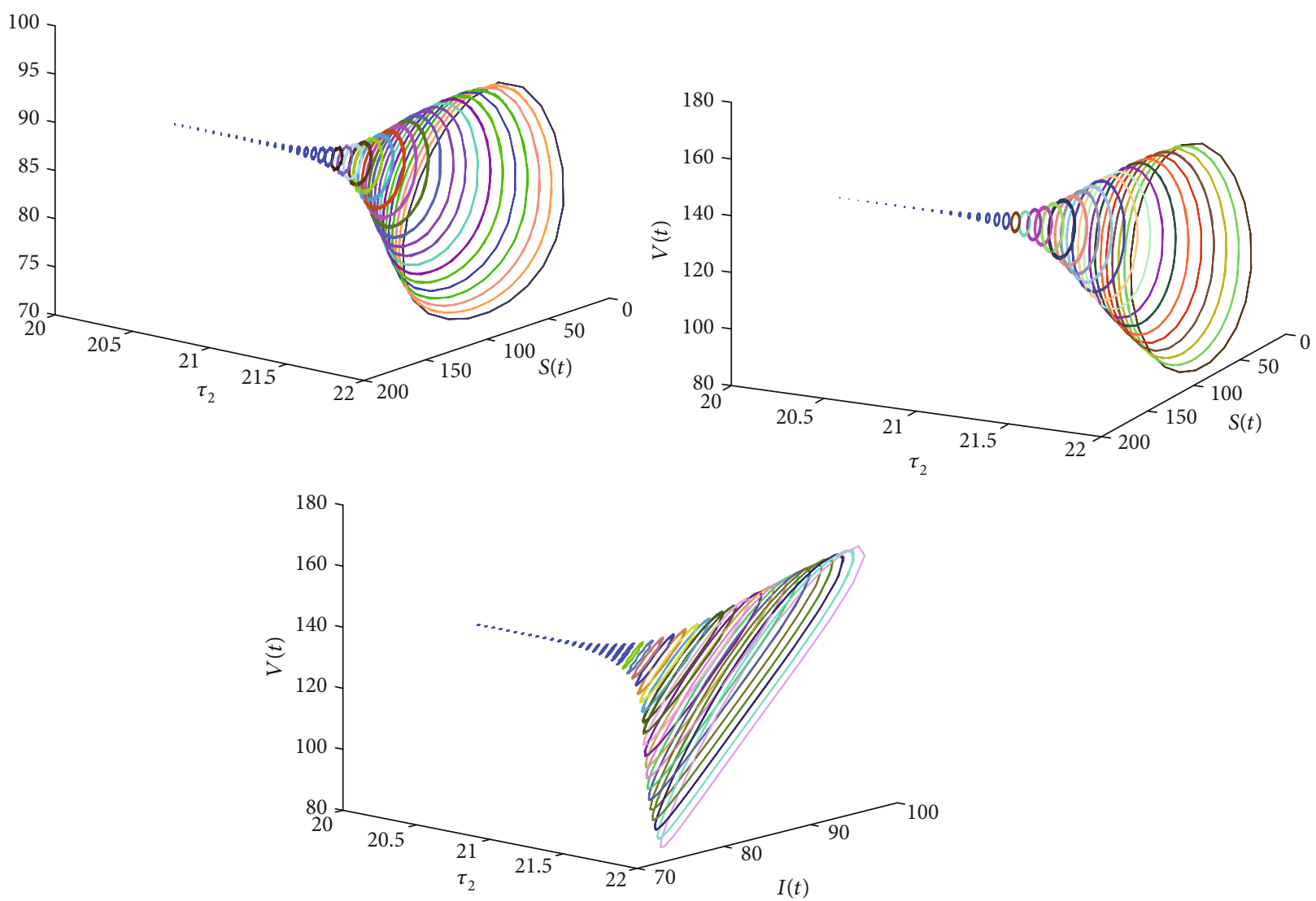

Figure 7: The bifurcation diagrams with $\tau_{2}$ as parameter and $\tau_{1}=23$. A Hopf bifurcation exists at $\tau_{2}=\tau_{2}^{*}$.

where $u_{t}(\theta)=u(t+\theta)=\left(u_{1}(t+\theta), \quad u_{2}(t+\theta), \quad u_{3}(t+\theta)\right), \theta$ $\in[-1,0]$.

For $\varphi \in C\left([0,1],\left(\mathbb{R}^{3}\right)^{*}\right)$, we define

$$
A^{*} \varphi(s)= \begin{cases}-\frac{\mathrm{d} \psi(s)}{d s}, & s \in(0,1], \\ \int_{-1}^{0} \mathrm{~d} \eta^{T}(t, 0) \varphi(-t), & s=0,\end{cases}
$$

and the bilinear inner product

$$
\langle\varphi(s), \phi(\theta)\rangle=\bar{\varphi}(0) \phi(0)-\int_{-1}^{0} \int_{\xi=0}^{\theta} \bar{\varphi}(\xi-\theta) d \eta(\theta) \phi(\xi) d \xi
$$

where $\eta(\theta)=\eta(\theta, 0)$. Let $A=A(0)$, then $A$ and $A^{*}$ are a pair of adjoint operators. From the discussions in Section 3, we know that $\pm i \omega_{2}^{*} \tau_{2}^{*}$ are a pair of eigenvalues of $A(0)$, it follows that they are also a pair of eigenvalues of $A^{*}$. Next, we calculate the eigenvectors of $A(0)$ and $A^{*}$ with respect to $i \omega_{2}^{*} \tau_{2}^{*}$ and $-i \omega_{2}^{*} \tau_{2}^{*}$, respectively.
Suppose that $q(\theta)$ is an eigenfunction of $A(0)$ corresponding to $i \omega_{2}^{*} \tau_{2}^{*}$. Then, by the definition of $A$, we can take

$$
q(\theta)=\left(1, q_{2}, q_{3}\right)^{T} e^{i \omega_{2}^{*} \tau_{2}^{*} \theta},
$$

where

$$
\begin{gathered}
q_{2}=\frac{\left(i \omega_{2}^{*}+\mu_{2}+b \beta_{2} I^{*} e^{-i \omega_{2}^{*} \tau_{2}^{*}}\right)\left(i \omega_{2}^{*}+\mu_{1}+b \beta_{1} V^{*} e^{-i \omega_{2}^{*} \tau_{1}^{*}}\right)}{-b \beta_{2}\left(\left(\Lambda_{2} / \mu_{2}\right)-V^{*}\right) e^{-i \omega_{2}^{*} \tau_{2}^{*}}}, \\
q_{3}=\frac{i \omega_{2}^{*}+\mu_{1}+b \beta_{1} V^{*} e^{-i \omega_{2}^{*} \tau_{1}^{*}}}{-b \beta_{1} S^{*} e^{-i \omega_{2}^{*} \tau_{1}^{*}}} .
\end{gathered}
$$

Similarly, $q^{*}(s)=D\left(1, q_{2}^{*}, q_{3}^{*}\right)^{T} e^{i \omega_{2}^{*} \tau_{2}^{*} s}, s \in[0,1]$ is the eigenfunction of $A^{*}(0)$ corresponding to $-i \omega_{2}^{*} \tau_{2}^{*}$, where

$$
\begin{gathered}
q_{2}^{*}=-\frac{-i \omega_{2}^{*}+\mu_{1}+b \beta_{1} V^{*} e^{i \omega_{2}^{*} \tau_{1}^{*}}}{\left(\sigma-e^{i \omega_{2}^{*} \tau_{1}^{*}}\right) b \beta_{1} V^{*}}, \\
q_{3}^{*}=\frac{\left(-i \omega_{2}^{*}+\mu_{1}+\gamma+b \sigma \beta_{1} V^{*}\right)\left(-i \omega_{2}^{*}+\mu_{1}+b \beta_{1} V^{*} e^{i \omega_{2}^{*} \tau_{1}^{*}}\right)}{b \beta_{2}\left(\left(\Lambda_{2} / \mu_{2}\right)-V^{*}\right) e^{i \omega_{2}^{*} \tau_{2}^{*}}\left(\sigma-e^{i \omega_{2}^{*} \tau_{1}^{*}}\right) b \beta_{1} V^{*}} .
\end{gathered}
$$


From equation (38), we can get

$$
\begin{aligned}
\left\langle q^{*}(s), q(\theta)\right\rangle= & \bar{q}^{*}(0) q(0)-\int_{-1}^{0} \int_{\xi=0}^{\theta} \bar{q}^{*}(\xi-\theta) d \eta(\theta) q(\xi) d \xi \\
= & \bar{D}\left(1, \bar{q}_{2}^{*}, \bar{q}_{3}^{*}\right)\left(1, q_{2}, q_{3}\right)^{T}-\bar{D} \int_{-1}^{0} \int_{\xi=0}^{\theta} \\
& \cdot\left(1, \bar{q}_{2}^{*}, \bar{q}_{3}^{*}\right) e^{i \omega_{2}^{*} \tau_{2}^{*}(\theta-\xi)} d \eta(\theta)\left(1, q_{2}, q_{3}\right)^{T} e^{i \omega_{2}^{*} \tau_{2}^{*} \xi} d \xi \\
= & \bar{D}\left[1+q_{2} \bar{q}_{2}^{*}+q_{3} \bar{q}_{3}^{*}-\left(1, \bar{q}_{2}^{*}, \bar{q}_{3}^{*}\right) \int_{-1}^{0} \theta e^{i \omega_{2}^{*} \tau_{2}^{*} \theta} d \eta(\theta)\left(1, q_{2}, q_{3}\right)^{T}\right] \\
= & \bar{D}\left[1+q_{2} \bar{q}_{2}^{*}+q_{3} \bar{q}_{3}^{*}+\tau_{1}^{*} b \beta_{1} e^{-i \omega_{2}^{*} \tau_{1}^{*}}\left(-V^{*}+\bar{q}_{2}^{*}-S^{*} q_{3}+\bar{q}_{2}^{*} q_{3}\right)\right. \\
& \left.+\tau_{2}^{*} b \beta_{2} e^{-i \omega_{2}^{*} \tau_{2}^{*}}\left(\left(\frac{\Lambda_{2}}{\mu_{2}}-V^{*}\right) q_{2}-I^{*} q_{3}\right) \bar{q}_{3}^{*}\right] .
\end{aligned}
$$

Thus, one can choose $D$ as

$$
\begin{aligned}
D= & {\left[1+\bar{q}_{2} q_{2}^{*}+\bar{q}_{3} q_{3}^{*}+\tau_{1}^{*} b \beta_{1} e^{i \omega_{2}^{*} \tau_{1}^{*}}\left(-V^{*}+\bar{q}_{2}^{*}-S^{*} q_{3}+\bar{q}_{2}^{*} q_{3}\right)\right.} \\
& \left.+\tau_{2}^{*} b \beta_{2} e^{i \omega_{2}^{*} \tau_{2}^{*}}\left(\left(\frac{\Lambda_{2}}{\mu_{2}}-V^{*}\right) q_{2}-I^{*} q_{3}\right) \bar{q}_{3}^{*}\right]^{-1},
\end{aligned}
$$

which satisfies $\left\langle q^{*}(s), q(\theta)\right\rangle=1$.

Next, using the methods given in [20], we can calculate some explicit expressions as follows that are used to determine the qualities of bifurcating periodic solution.

$$
\begin{aligned}
g_{20}= & 2 \bar{D} \tau_{2}^{*}\left[-b \beta_{1} q_{3} e^{-2 i \omega_{2}^{*} \tau_{1}^{*}}+\bar{q}_{2}^{*}\left(b \beta_{1} q_{3} e^{-2 i \omega_{2}^{*} \tau_{1}^{*}}-\sigma b \beta_{1} q_{3}-\sigma b \beta_{1} q_{2} q_{3}\right)\right. \\
& \left.-\bar{q}_{3}^{*} b \beta_{2} q_{2} q_{3} e^{-2 i \omega_{2}^{*} \tau_{2}^{*}}\right]
\end{aligned}
$$

$$
\begin{aligned}
& g_{11}=\bar{D} \tau_{2}^{*}\left[-b \beta_{1}\left(\bar{q}_{3}+q_{3}\right)+\bar{q}_{2}^{*}\left((1-\sigma) b \beta_{1}\left(\bar{q}_{3}+q_{3}\right)\right.\right. \\
& \left.\left.-b \sigma \beta_{1}\left(q_{2} \bar{q}_{3}+\bar{q}_{2} q_{3}\right)\right)-\bar{q}_{3}^{*} b \beta_{2}\left(q_{2} \bar{q}_{3}+\bar{q}_{2} q_{3}\right)\right] \text {, } \\
& g_{02}=2 \bar{D} \tau_{2}^{*}\left[-b \beta_{1} \bar{q}_{3} e^{2 i \omega_{2}^{*} \tau_{1}^{*}}+\bar{q}_{2}^{*}\left(b \beta_{1} \bar{q}_{3} e^{2 i \omega_{2}^{*} \tau_{1}^{*}}-\sigma b \beta_{1} \bar{q}_{3}-\sigma b \beta_{1} \bar{q}_{2} \bar{q}_{3}\right)\right. \\
& \left.-\bar{q}_{3}^{*} b \beta_{2} \bar{q}_{2} \bar{q}_{3} e^{2 i \omega_{2}^{*} \tau_{2}^{*}}\right] \\
& g_{21}=\bar{D} \tau_{2}^{*}\left\{-b \beta_{1}\left[2 W_{11}^{(3)}\left(-\frac{\tau_{1}^{*}}{\tau_{2}^{*}}\right) e^{-i \omega_{2}^{*} \tau_{1}^{*}}+W_{20}^{(3)}\left(-\frac{\tau_{1}^{*}}{\tau_{2}^{*}}\right) e^{i \omega_{2}^{*} \tau_{1}^{*}}\right.\right. \\
& \left.+W_{20}^{(1)}\left(-\frac{\tau_{1}^{*}}{\tau_{2}^{*}}\right) \bar{q}_{3} e^{i \omega_{2}^{*} \tau_{1}^{*}}+2 W_{11}^{(1)}\left(-\frac{\tau_{1}^{*}}{\tau_{2}^{*}}\right) q_{3} e^{-i \omega_{2}^{*} \tau_{1}^{*}}\right] \\
& +\bar{q}_{2}^{*}\left[b \beta _ { 1 } \left(2 W_{11}^{(3)}\left(-\frac{\tau_{1}^{*}}{\tau_{2}^{*}}\right) e^{-i \omega_{2}^{*} \tau_{1}^{*}}+W_{20}^{(3)}\left(-\frac{\tau_{1}^{*}}{\tau_{2}^{*}}\right) e^{i \omega_{2}^{*} \tau_{1}^{*}}\right.\right. \\
& \left.+W_{20}^{(1)}\left(-\frac{\tau_{1}^{*}}{\tau_{2}^{*}}\right) \bar{q}_{3} e^{i \omega_{2}^{*} \tau_{1}^{*}}+2 W_{11}^{(1)}\left(-\frac{\tau_{1}^{*}}{\tau_{2}^{*}}\right) q_{3} e^{-i \omega_{2}^{*} \tau_{1}^{*}}\right) \\
& -\sigma b \beta_{1}\left(2 W_{11}^{(3)}(0)+W_{20}^{(3)}(0)+W_{20}^{(1)}(0) \bar{q}_{3}+2 W_{11}^{(1)}(0) q_{3}\right) \\
& \left.-b \sigma \beta_{1}\left(2 W_{11}^{(3)}(0) q_{2}+W_{20}^{(3)}(0) \bar{q}_{2}+W_{20}^{(2)}(0) \bar{q}_{3} 2 W_{11}^{(2)}(0) q_{3}\right)\right] \\
& -\bar{q}_{3}^{*} b \beta_{2}\left[2 W_{11}^{(3)}(-1) q_{2} e^{-i \omega_{2}^{*} \tau_{2}^{*}}+W_{20}^{(3)}(-1) \bar{q}_{2} e^{i \omega_{2}^{*} \tau_{2}^{*}}\right. \\
& \left.\left.+W_{20}^{(2)}(-1) \bar{q}_{3} e^{i \omega_{2}^{*} \tau_{2}^{*}}+2 W_{11}^{(2)}(-1) q_{3} e^{-i \omega_{2}^{*} \tau_{2}^{*}}\right]\right\},
\end{aligned}
$$

where

$$
\begin{aligned}
& W_{20}(\theta)=\frac{i g_{20}}{\omega_{2}^{*} \tau_{2}^{*}} q(0) e^{i \omega_{2}^{*} \tau_{2}^{*} \theta}+\frac{i \bar{g}_{02}}{3 \omega_{2}^{*} \tau_{2}^{*}} \bar{q}(0) e^{-i \omega_{2}^{*} \tau_{2}^{*} \theta}+E_{1} e^{2 i \omega_{2}^{*} \tau_{2}^{*} \theta} \\
& W_{11}(\theta)=-\frac{i g_{11}}{\omega_{2}^{*} \tau_{2}^{*}} q(0) e^{i \omega_{2}^{*} \tau_{2}^{*} \theta}+\frac{i \bar{g}_{11}}{\omega_{2}^{*} \tau_{2}^{*}} \bar{q}(0) e^{-i \omega_{2}^{*} \tau_{2}^{*} \theta}+E_{2}, \\
& E_{1}=2\left(\frac{\Delta_{11}}{\Delta_{1}}, \frac{\Delta_{12}}{\Delta_{1}}, \frac{\Delta_{13}}{\Delta_{1}}\right) \text {, } \\
& E_{2}=\left(\frac{\Delta_{21}}{\Delta_{2}}, \frac{\Delta_{22}}{\Delta_{2}}, \frac{\Delta_{23}}{\Delta_{2}}\right) \text {, } \\
& \Delta_{1}=\left|\begin{array}{ccc}
2 i \omega_{2}^{*}+\mu_{1}+b \beta_{1} V^{*} e^{-2 i \omega_{2}^{*} \tau_{1}^{*}} & 0 & b \beta_{1} S^{*} e^{-2 i \omega_{2}^{*} \tau_{1}^{*}} \\
\left(\sigma-e^{-2 i \omega_{2}^{*} \tau_{1}^{*}}\right) b \beta_{1} V^{*} & 2 i \omega_{2}^{*}+\mu_{1}+\gamma+b \sigma \beta_{1} V^{*} & -b \beta_{1} S^{*} e^{-2 i \omega_{2}^{*} \tau_{1}^{*}}-b \sigma \beta_{1}\left(\frac{\Lambda_{1}}{\mu_{1}}-I^{*}-S^{*}\right) \\
0 & -b \beta_{2}\left(\frac{\Lambda_{2}}{\mu_{2}}-V^{*}\right) e^{-2 i \omega_{2}^{*} \tau_{2}^{*}} & 2 i \omega_{2}^{*}+\mu_{2}+b \beta_{2} I^{*} e^{-2 i \omega_{2}^{*} \tau_{2}^{*}}
\end{array}\right|, \\
& \Delta_{2}=\left|\begin{array}{ccc}
\mu_{1}+b \beta_{1} V^{*} & 0 & b \beta_{1} S^{*} \\
-(1-\sigma) b \beta_{1} V^{*} & \mu_{1}+\lambda+b \sigma \beta_{1} V^{*} & -\frac{\left(\mu_{1}+\gamma\right) I^{*}}{V^{*}} \\
0 & -b \beta_{2}\left(\frac{\Lambda_{2}}{\mu_{2}}-V^{*}\right) & \mu_{2}+b \beta_{2} I^{*}
\end{array}\right|
\end{aligned}
$$



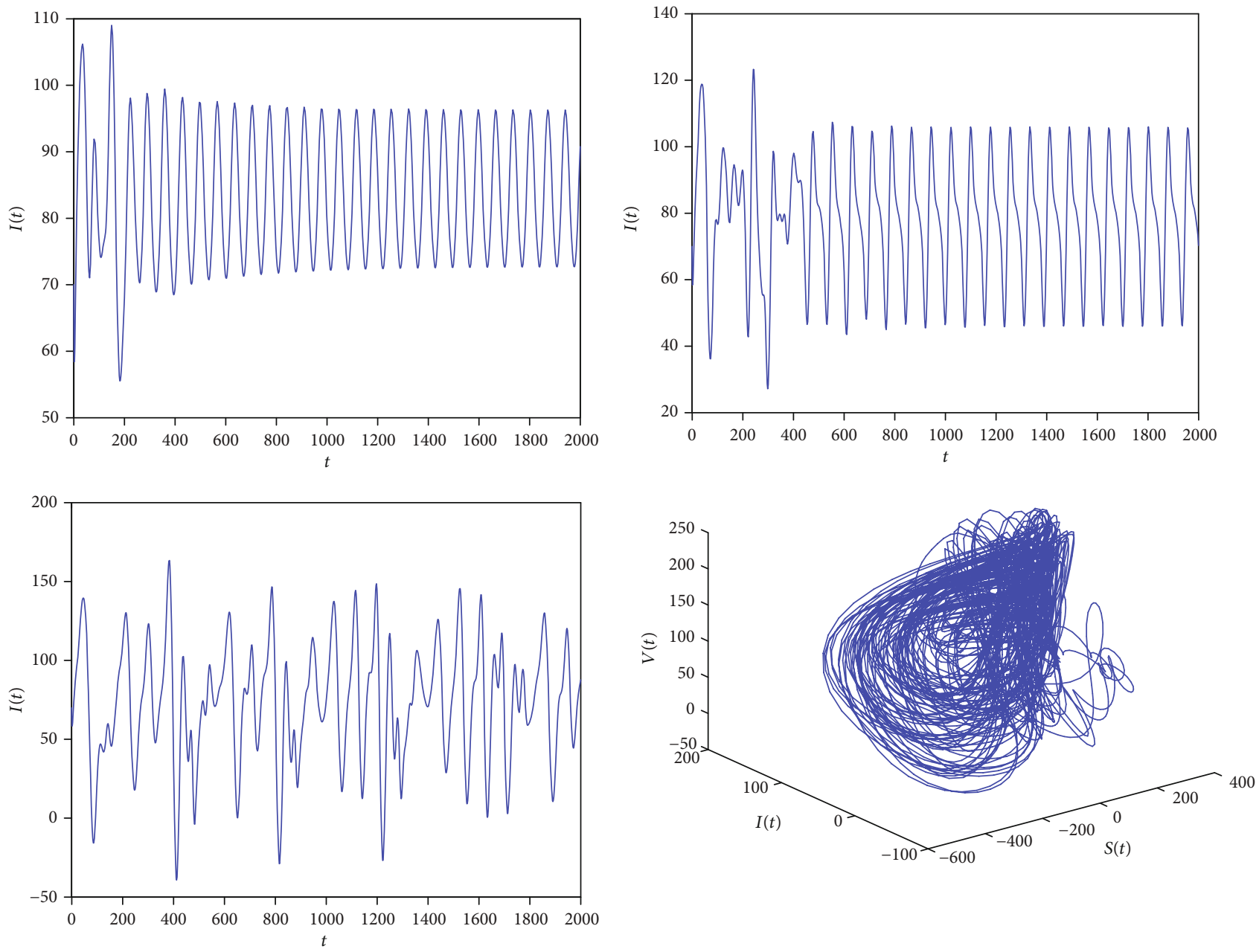

Figure 8: The bifurcation periodic solutions of $I(t)$ with $\tau_{1}=23$ and $\tau_{2}=22,28,38$ (from the top to the first of the bottom). The bifurcation periodic solution is unstable with $\tau_{1}=23$ and $\tau_{2}=38$.

$\Delta_{11}, \Delta_{12}$, and $\Delta_{13}$ are the third-order determinants obtained by substituting the first, second, and third columns of $\Delta_{1}$ by vector $\left(P_{1}, P_{2}, P_{3}\right)^{T}$, respectively. $\Delta_{21}, \Delta_{22}$, and $\Delta_{23}$ are also third-order determinants obtained by replacing the first, second, and third columns of $\Delta_{2}$ by vector $\left(Q_{1}, Q_{2}, Q_{3}\right)^{T}$, respectively, where

$$
\begin{gathered}
P_{1}=-b \beta_{1} q_{3} e^{2 i \omega_{2}^{*} \tau_{1}^{*}}, \\
P_{2}=b \beta_{1} q_{3} e^{-2 i \omega_{2}^{*} \tau_{1}^{*}}-\sigma b \beta_{1} q_{3}-\sigma b \beta_{1} q_{2} q_{3}, \\
P_{3}=-b \beta_{2} q_{2} q_{3} e^{-2 i \omega_{2}^{*} \tau_{2}^{*},} \\
Q_{1}=-b \beta_{1} \operatorname{Re}\left\{q_{3}\right\}, \\
Q_{2}=(1-\sigma) b \beta_{1} \operatorname{Re}\left\{q_{3}\right\}-b \sigma \beta_{1} \operatorname{Re}\left\{q_{2} \bar{q}_{3}\right\}, \\
Q_{3}=-b \beta_{2} \operatorname{Re}\left\{q_{2} \bar{q}_{3}\right\} .
\end{gathered}
$$

Thus, the following quantities can be calculated:

$$
\left\{\begin{array}{l}
c_{1}(0)=\frac{i}{2 \omega_{2}^{*} \tau_{2}^{*}}\left(g_{20} g_{11}-2\left|g_{11}\right|^{2}-\frac{1}{3}\left|g_{02}\right|^{2}\right)+\frac{1}{2} g_{21} \\
\mu_{2}=-\frac{\operatorname{Re}\left\{c_{1}(0)\right\}}{\operatorname{Re}\left\{\lambda^{\prime}\left(\tau_{2}^{*}\right)\right\}} \\
\beta_{2}=2 \operatorname{Re}\left\{c_{1}(0)\right\} \\
T_{2}=-\frac{1}{\omega_{2}^{*} \tau_{2}^{*}}\left(\operatorname{Im}\left\{c_{1}(0)\right\}+\mu_{2} \operatorname{Im}\left\{\lambda^{\prime}\left(\tau_{2}^{*}\right)\right\}\right)
\end{array}\right.
$$

Therefore, based on [20], we can obtain the main results in the present section as follows.

Theorem 6. For system (2), the periodic solution is supercritical (resp., subcritical) if $\mu_{2}>0$ (resp., $\left.\mu_{2}<0\right)$. The bifurcating periodic solutions are orbitally asymptotically stable with an 

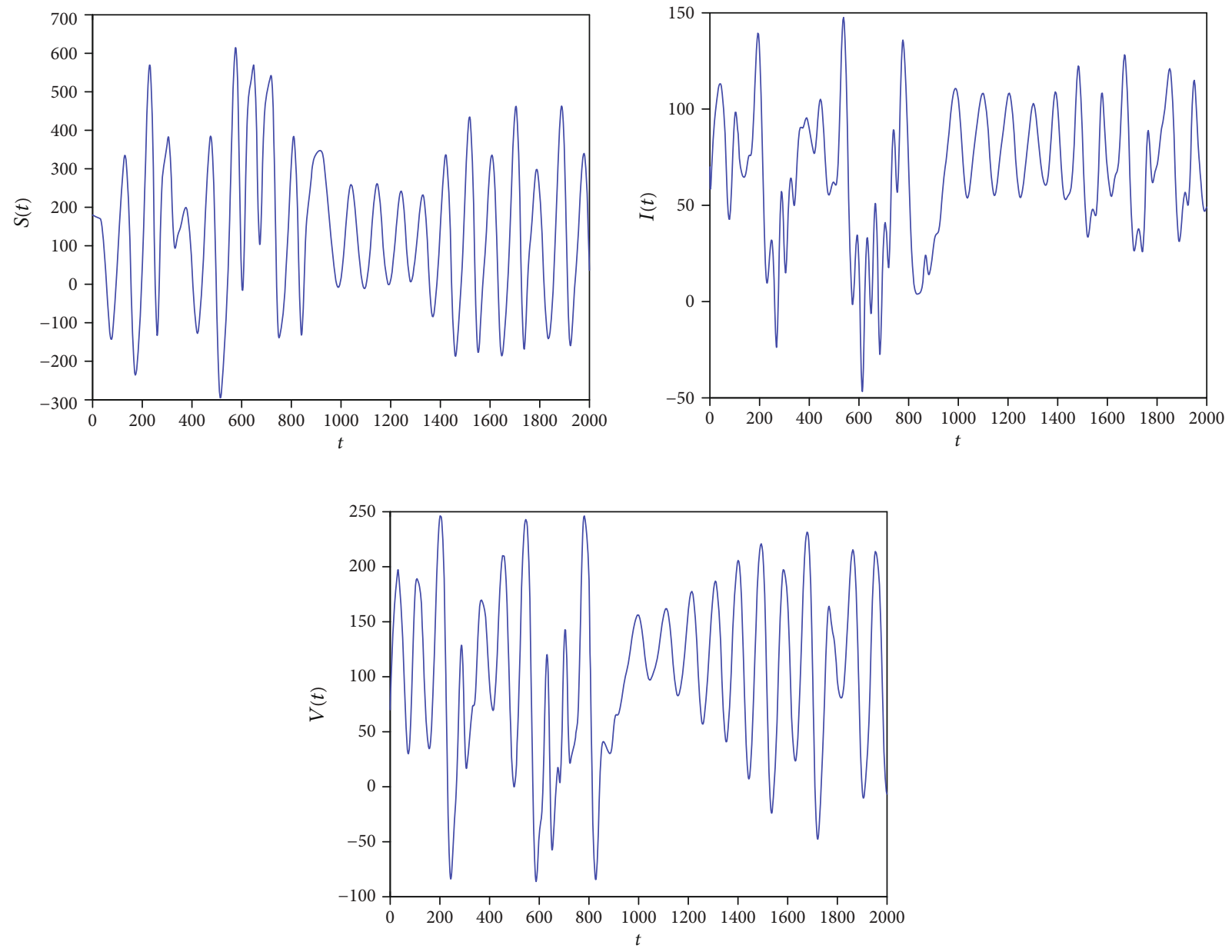

FIgURE 9: The system appears irregular periodic behaviors when $\tau_{1}=31$ and $\tau_{2}=31$.

asymptotical phase (resp., unstable) if $\beta_{2}<0$ (resp., $\beta_{2}>0$ ). The period of the bifurcating periodic solutions increases (resp., decreases) if $T_{2}>0$ (resp. $\left.T_{2}<0\right)$.

\section{Numerical Examples and Simulations}

We select the parameters to simulate the above theoretical results as follows:

$$
\begin{gathered}
\Lambda_{1}=9, b=0.3, \\
\beta_{1}=0.002, \mu_{1}=0.01, \\
\sigma=0.8, \gamma=0.6, \\
\Lambda_{2}=9, \\
\beta_{2}=0.005, \\
\mu_{2}=0.05 .
\end{gathered}
$$

By calculations, we can obtain that the endemic equilibrium of system (2) is $E^{*}=\left(S^{*}, I^{*}, V^{*}\right)=(102.5734,85.6437$ , 129.5701). Under the above parameters, the condition (H1) is satisfied. According to Theorem 2, we know that
TABLE 1: The relationships among the partial immunity $\sigma$, the endemic equilibrium $E^{*}$, and the critical values $\tau_{10}$ and $\tau_{2}^{*}$.

\begin{tabular}{lccccc}
\hline$\sigma$ & 1 & 0.8 & 0.6 & 0.4 & 0.2 \\
\hline$S^{*}$ & 97.4478 & 102.5734 & 111.3948 & 129.1222 & 170.4044 \\
$I^{*}$ & 107.0566 & 85.6437 & 63.4246 & 41.2030 & 21.8956 \\
$V^{*}$ & 137.2619 & 129.5701 & 117.9896 & 99.5023 & 71.3613 \\
$\tau_{10}$ & 21.5895 & 23.3291 & 26.6112 & 34.3647 & 56.7849 \\
$\tau_{2}^{*}$ & 17.8120 & 21.4739 & 25.5925 & 30.6267 & 44.0800 \\
\hline
\end{tabular}

the endemic equilibrium $E^{*}$ of system (2) is asymptotically stable when $\tau_{1}=\tau_{2}=0$ (see Figure 1). When $\tau_{1}>0, \tau_{2}=0$, we get $p_{0}=-4.7847 \times 10^{-5}<0, \tau_{10}=23.3291, z_{1}=0.0053$, and $f^{\prime}\left(z_{1}\right)=0.017 \neq 0$; from Theorem 4 , the endemic equilibrium $E^{*}$ is locally asymptotically stable for $\tau_{1} \in\left(0, \tau_{10}\right)$ and is unstable for $\tau_{1}>\tau_{10}$, and a Hopf bifurcation occurs at $\tau_{1}=\tau_{10}$ (see Figures 2-4). When $\tau_{2}>0$, fixing $\tau_{1}=23$ that belongs to its stable interval $\left(0, \tau_{10}\right)$, then, by calculation, we obtain that a positive root of equation (22) is $\omega=0.0933$ and $\tau_{2}^{*}=21.4739$, which follow from Theorem 5 that the endemic equilibrium $E^{*}$ is locally asymptotically stable for $\tau_{2} \in\left(0, \tau_{2}^{*}\right)$ 
and is unstable for $\tau_{2} \in\left(\tau_{2}^{*},+\infty\right)$, a Hopf bifurcation exits at $\tau_{2}=\tau_{2}^{*}$ (see Figures 5-7).

The numerical results show that $c_{1}(0)=1.0266 \times 10^{-5}-$ $4.7626 \times 10^{-6} i, \mu_{2}=-0.0024<0, \beta_{2}=2.0531 \times 10^{-5}>0$, and $T_{2}=2.3115 \times 10^{-6}>0$, which imply that, for system (2), its periodic solution is subcritical at $\tau_{2}^{*}$ and unstable, and the period of the bifurcating periodic solutions increases (see Figure 8). Also, when the two delays are greater than their critical values, the system will produce irregular periodic behaviors, which is depicted in Figure 9.

Besides, the influences of the parameter $\sigma$ on the endemic equilibrium $E^{*}$ and the critical values $\tau_{10}$ and $\tau_{2}^{*}$ of the two delays $\tau_{1}$ and $\tau_{2}$ are shown in Table 1 . When $\sigma$ decreases, $I^{*}$ and $V^{*}$ also decrease, but $S^{*}, \tau_{10}$, and $\tau_{2}^{*}$ increase, which suggests that the degree of partial protection for recovered people acquired by a primary infection can change the densities of distributions of susceptible and infective people and can also affect on the stability of positive equilibrium. It is noteworthy that it can not change the direction of Hopf bifurcation and the stability of bifurcating periodic solutions.

\section{Conclusions}

In this paper, we considered a vector-borne disease model with two delays and reinfection based on the models proposed by [4-6]. Some theoretical analyses of this model were implemented. From the characteristic equations, firstly, we discussed the conditions of the endemic equilibrium $E^{*}$, which is locally asymptotically stable in the model without delays by the Routh-Hurwitz criterion. Then, by setting $\tau_{2}$ $=0$, regarding $\tau_{1}$ as a bifurcating parameter, and setting $\tau_{1}$ in its stable interval, regarding $\tau_{2}$ as a parameter, respectively, we employed the Hopf bifurcation theorem to analyze the conditions for the stability of equilibrium and existence of Hopf bifurcation. Furthermore, when $\tau_{1}$ is in its stable interval, for parameter $\tau_{2}$, the directions and stability of Hopf bifurcation were investigated, and some explicit formulas were derived by using the normal form theory and center manifold theorem. In the end, our numerical examples and simulations verified the correctness of theoretical conclusions. As the facts show, the disease will be persistent under the set of parameters, and the number of the host population and vector population will fluctuate. Also, when both delays exceed their critical values, the stability of the system and the properties and behaviors of the bifurcating periodic solutions may lead to change. The oscillatory behavior and phenomenon that an increased partial immunity may give rise to instability will prompt medical workers engaged in infectious diseases to remain vigilant against the virus even if they have noticed that the number of infected populations decreases. The qualitative analysis and numerical simulation used in the research of dynamic behavior of system can provide theoretical basis for revealing the development process, transmission pattern, and change trend of diseases and give important reference to the analysis, prediction, and control of human infectious diseases.

\section{Appendix}

\section{A. The Derivation Process of $R_{0}$ Based on the Next-Generation Method}

$R_{0}$ is the dominant eigenvalue of the next generation matrix $G=F V^{-1}$ whose entries are interpreted as the number of secondary infections produced by infected vectors and hosts in the process of their infection. Here, the notation we use follows [22-24]. For system (2), we calculate

$$
\begin{gathered}
F=\left(\begin{array}{cc}
0 & \frac{b \beta_{1} \Lambda_{1}}{\mu_{1}} \\
\frac{b \beta_{2} \Lambda_{2}}{\mu_{2}} & 0
\end{array}\right), \\
V=\left(\begin{array}{cc}
\mu_{1}+\gamma & 0 \\
0 & \mu_{2}
\end{array}\right), \\
G=\left(\begin{array}{cc}
0 \beta_{1} \Lambda_{1} \\
\mu_{1} \mu_{2} \\
\frac{b \beta_{2} \Lambda_{2}}{\left(\mu_{1}+\gamma\right) \mu_{2}} & 0
\end{array}\right) .
\end{gathered}
$$

Thus, $R_{0}=\left(b^{2} \Lambda_{1} \Lambda_{2} \beta_{1} \beta_{2}\right) /\left(\mu_{1} \mu_{2}^{2}\left(\mu_{1}+\gamma\right)\right)$. Note that we use $R_{0}^{2}$ as in [5], though it is called $R_{0}$. Either way, its threshold behavior $R_{0}>1$ remains unchanged.

\section{B. The Derivation Process of Coefficients $g_{20}$, $g_{11}, g_{02}$, and $g_{21}$ of Equation (47)}

Using the algorithms and marks presented in [20], when $\mu$ $=0$, the projection system of system (2) on the center manifold $C_{0}$ can be calculated. For the solution $u_{t}$ of equation (29), we define $z(t)=\left\langle q^{*}, u_{t}\right\rangle$, which follows from equations (35)-(39) that

$$
\dot{z}(t)=\left\langle q^{*}, \dot{u}_{t}\right\rangle=\left\langle A^{*}(0) q^{*}, u_{t}\right\rangle+\bar{q}^{*}(0) F\left(0, u_{t}\right) \triangleq i \omega_{2}^{*} \tau_{2}^{*} z+g(z, \bar{z}),
$$

where $g(z, \bar{z})=\bar{q}^{*}(0) F\left(0, u_{t}\right)=g_{20}(\theta)\left(z^{2} / 2\right)+g_{11}(\theta) z \bar{z}+$ $g_{02}(\theta)\left(\bar{z}^{2} / 2\right)+g_{21}(\theta)\left(z^{2} \bar{z} / 2\right) \cdots$.

Let

$$
W(t, \theta)=u_{t}(\theta)-2 \operatorname{Re}\{z(t) q(\theta)\}
$$

then, on the center manifold $C_{0}$, we can have

$$
W(t, \theta)=W(z(t), \bar{z}(t), \theta),
$$

where

$$
W(z, \bar{z}, \theta)=W_{20}(\theta) \frac{z^{2}}{2}+W_{11}(\theta) z \bar{z}+W_{02}(\theta) \frac{\bar{z}^{2}}{2}+W_{30}(\theta) \frac{z^{3}}{6}+\cdots
$$

$z$ and $\bar{z}$ are local coordinates on $C_{0}$ in the directions of $q^{*}$ 
and $\bar{q}^{*}$. From equations (39) and (B.2), we can see

$$
\begin{aligned}
u_{t}(\theta)= & W(t, \theta)+z(t) q(\theta)+\bar{z}(t) \bar{q}(\theta)=\left(1, q_{2}, q_{3}\right)^{T} e^{i \omega_{2}^{*} \tau_{2}^{*} \theta} z \\
& +\left(1, \bar{q}_{2}, \bar{q}_{3}\right)^{T} e^{-i \omega_{2}^{*} \tau_{2}^{*} \theta} \bar{z}+W_{20}(\theta) \frac{z^{2}}{2}+W_{11}(\theta) z \bar{z} \\
& +W_{02}(\theta) \frac{\bar{z}^{2}}{2}+W_{30}(\theta) \frac{z^{3}}{6}+\cdots
\end{aligned}
$$

Therefore, we can obtain

$$
\begin{aligned}
& u_{1 t}(0)=z+\bar{z}+W_{20}^{(1)}(0) \frac{z^{2}}{2}+W_{11}^{(1)}(0) z \bar{z}+W_{02}^{(1)}(0) \frac{\bar{z}^{2}}{2}+\cdots \\
& u_{2 t}(0)=z q_{2}+\bar{z} \bar{q}_{2}+W_{20}^{(2)}(0) \frac{z^{2}}{2}+W_{11}^{(2)}(0) z \bar{z}+W_{02}^{(2)}(0) \frac{\bar{z}^{2}}{2}+\cdots \\
& u_{3 t}(0)=z q_{3}+\bar{z} \bar{q}_{3}+W_{20}^{(3)}(0) \frac{z^{2}}{2}+W_{11}^{(3)}(0) z \bar{z}+W_{02}^{(3)}(0) \frac{\bar{z}^{2}}{2}+\cdots, \\
& u_{1 t}(-1)=z e^{-i \omega_{2}^{*} \tau_{2}^{*}}+\bar{z} e^{i \omega_{2}^{*} \tau_{2}^{*}}+W_{20}^{(1)}(-1) \frac{z^{2}}{2}+W_{11}^{(1)}(-1) z \bar{z} \\
& +W_{02}^{(1)}(-1) \frac{\bar{z}^{2}}{2}+\cdots \\
& u_{2 t}(-1)=z q_{2} e^{-i \omega_{2}^{*} \tau_{2}^{*}}+\bar{z} \bar{q}_{2} e^{i \omega_{2}^{*} \tau_{2}^{*}}+W_{20}^{(2)}(-1) \frac{z^{2}}{2}+W_{11}^{(2)}(-1) z \bar{z} \\
& +W_{02}^{(2)}(-1) \frac{\bar{z}^{2}}{2}+\cdots,
\end{aligned}
$$

$$
\begin{aligned}
u_{2 t}\left(-\frac{\tau_{1}^{*}}{\tau_{2}^{*}}\right)= & z q_{2} e^{-i \omega_{2}^{*} \tau_{1}^{*}}+\bar{z} \bar{q}_{2} e^{i \omega_{2}^{*} \tau_{1}^{*}}+W_{20}^{(2)}\left(-\frac{\tau_{1}^{*}}{\tau_{2}^{*}}\right) \frac{z^{2}}{2} \\
& +W_{11}^{(2)}\left(-\frac{\tau_{1}^{*}}{\tau_{2}^{*}}\right) z \bar{z}+W_{02}^{(2)}\left(-\frac{\tau_{1}^{*}}{\tau_{2}^{*}}\right) \frac{\bar{z}^{2}}{2}+\cdots \\
u_{3 t}\left(-\frac{\tau_{1}^{*}}{\tau_{2}^{*}}\right)= & z q_{3} e^{-i \omega_{2}^{*} \tau_{1}^{*}}+\bar{z}_{\bar{q}_{3}} e^{i \omega_{2}^{*} \tau_{1}^{*}}+W_{20}^{(3)}\left(-\frac{\tau_{1}^{*}}{\tau_{2}^{*}}\right) \frac{z^{2}}{2} \\
& +W_{11}^{(3)}\left(-\frac{\tau_{1}^{*}}{\tau_{2}^{*}}\right) z \bar{z}+W_{02}^{(3)}\left(-\frac{\tau_{1}^{*}}{\tau_{2}^{*}}\right) \frac{\bar{z}^{2}}{2}+\cdots
\end{aligned}
$$

Furthermore, we can get $g(z, \bar{z})$ as follows:

$$
\begin{aligned}
g(z, \bar{z})= & \bar{q}^{*}(0) F\left(0, u_{t}\right)=\bar{D}\left(1, \bar{q}_{2}^{*}, \bar{q}_{3}^{*}\right) F\left(0, u_{t}\right)=\bar{D}\left(1, \bar{q}_{2}^{*}, \bar{q}_{3}^{*}\right) \tau_{2}^{*}\left(\begin{array}{r}
-b \beta_{1} u_{1 t}\left(-\frac{\tau_{1}^{*}}{\tau_{2}^{*}}\right) u_{3 t}\left(-\frac{\tau_{1}^{*}}{\tau_{2}^{*}}\right) \\
b \beta_{1} u_{1 t}\left(-\frac{\tau_{1}^{*}}{\tau_{2}^{*}}\right) u_{3 t}\left(-\frac{\tau_{1}^{*}}{\tau_{2}^{*}}\right)-\sigma b \beta_{1} u_{1 t}(0) u_{3 t}(0)-b \sigma \beta_{1} u_{2 t}(0) u_{3 t}(0) \\
-b \beta_{2} u_{2 t}(-1) u_{3 t}(-1)
\end{array}\right) \\
= & \bar{D} \tau_{2}^{*}\left\{-b \beta_{1} u_{1 t}\left(-\frac{\tau_{1}^{*}}{\tau_{2}^{*}}\right) u_{3 t}\left(-\frac{\tau_{1}^{*}}{\tau_{2}^{*}}\right)+\bar{q}_{2}^{*}\left[b \beta_{1} u_{1 t}\left(-\frac{\tau_{1}^{*}}{\tau_{2}^{*}}\right) u_{3 t}\left(-\frac{\tau_{1}^{*}}{\tau_{2}^{*}}\right)-\sigma b \beta_{1} u_{1 t}(0) u_{3 t}(0)-b \sigma \beta_{1} u_{2 t}(0) u_{3 t}(0)\right]\right. \\
& \left.-\bar{q}_{3}^{*} b \beta_{2} u_{2 t}(-1) u_{3 t}(-1)\right\} .
\end{aligned}
$$

Substituting $u_{1 t}(0), u_{2 t}(0), u_{3 t}(0), u_{2 t}(-1), u_{3 t}(-1), u_{1 t}($ $\left.-\tau_{1}^{*} / \tau_{2}^{*}\right)$, and $u_{3 t}\left(-\tau_{1}^{*} / \tau_{2}^{*}\right)$ into the above expression of $g(z$, $\bar{z})$ and comparing the coefficients yield the coefficients $g_{20}$, $g_{11}, g_{02}$, and $g_{21}$ of equation (47).

Obviously, $g_{20}, g_{11}$, and $g_{02}$ can be determined directly by substituting system parameters, but $W_{20}(\theta)$ and $W_{11}(\theta)$ in $g_{21}$ need to be calculated through some methods $[25,26]$.
By equations (36) and (B.2), we can get

$$
\begin{aligned}
\dot{W} & =\dot{u}_{t}-\dot{z} q-\dot{\bar{z}} \bar{q}=\left\{\begin{array}{l}
A W-2 \operatorname{Re}\left\{\bar{q}^{*}(0) F\left(0, u_{t}\right) q(\theta)\right\}, \theta \in[-1,0), \\
A W-2 \operatorname{Re}\left\{\bar{q}^{*}(0) F\left(0, u_{t}\right) q(0)\right\}+F\left(0, u_{t}\right), \theta=0,
\end{array}\right. \\
& \triangleq A W+H(z, \bar{z}, \theta),
\end{aligned}
$$


where

$$
H(z, \bar{z}, \theta)=H_{20}(\theta) \frac{z^{2}}{2}+H_{11}(\theta) z \bar{z}+H_{02}(\theta) \frac{\bar{z}^{2}}{2}+\cdots
$$

Thus, we have

$$
A W(t, \theta)-\dot{W}=-H(z, \bar{z}, \theta)=-H_{20}(\theta) \frac{z^{2}}{2}-H_{11}(\theta) z \bar{z}-H_{02}(\theta) \frac{\bar{z}^{2}}{2}-\cdots .
$$

From equation (B.4), we can get

$$
\begin{gathered}
A W(t, \theta)=A W_{20}(\theta) \frac{z^{2}}{2}+A W_{11}(\theta) z \bar{z}+A W_{02}(\theta) \frac{\bar{z}^{2}}{2}+A W_{30}(\theta) \frac{z^{3}}{6}+\cdots, \\
\dot{W}=W_{20}(\theta) z \dot{z}+W_{11}(\theta)(\dot{z} \bar{z}+z \dot{\bar{z}})+\cdots=2 i \omega^{*} \tau_{2}^{*} W_{20}(\theta) \frac{z^{2}}{2}+\cdots
\end{gathered}
$$

Therefore, we can obtain

$$
\left(A-2 i \omega^{*} \tau_{2}^{*}\right) W_{20}(\theta)=-H_{20}(\theta), A W_{11}(\theta)=-H_{11}(\theta) .
$$

For $\theta \in[-1,0)$, we have

$$
H(z, \bar{z}, \theta)=-g(z, \bar{z}) q(\theta)-\bar{g}(z, \bar{z}) \bar{q}(\theta) .
$$
get

By comparing coefficients of (B.9) with (B.13), one can

$$
\begin{gathered}
H_{20}(\theta)=-\left(g_{20} q(\theta)+\bar{g}_{02} \bar{q}(\theta)\right), \\
H_{11}(\theta)=-\left(g_{11} q(\theta)+\bar{g}_{11} \bar{q}(\theta)\right) .
\end{gathered}
$$

From equations (B.12) and (B.14) and the definition of $A$, we can obtain

$$
\dot{W}_{20}(\theta)=2 i \omega_{2}^{*} \tau_{2}^{*} W_{20}(\theta)+g_{20} q(\theta)+\bar{g}_{02} \bar{q}(\theta) .
$$

Since $q(\theta)=q(0) e^{i \omega_{2}^{*} \tau_{2}^{*} \theta}=\left(1, q_{2}, q_{3}\right)^{T} e^{i \omega_{2}^{*} \tau_{2}^{*} \theta}$, then according to (B.16), we can get

$$
W_{20}(\theta)=\frac{i g_{20}}{\omega_{2}^{*} \tau_{2}^{*}} q(0) e^{i \omega_{2}^{*} \tau_{2}^{*} \theta}+\frac{i \bar{g}_{02}}{3 \omega_{2}^{*} \tau_{2}^{*}} \bar{q}(0) e^{-i \omega_{2}^{*} \tau_{2}^{*} \theta}+E_{1} e^{2 i \omega_{2}^{*} \tau_{2}^{*} \theta},
$$

where $E_{1}=\left(E_{1}^{(1)}, E_{1}^{(2)}, E_{1}^{(3)}\right) \in \mathbb{R}^{3}$.
Similarly,

$$
\dot{W}_{11}(\theta)=g_{11} q(\theta)+\bar{g}_{11} \bar{q}(\theta)
$$

$$
W_{11}(\theta)=-\frac{i g_{11}}{\omega_{2}^{*} \tau_{2}^{*}} q(0) e^{i \omega_{2}^{*} \tau_{2}^{*} \theta}+\frac{i \bar{g}_{11}}{\omega_{2}^{*} \tau_{2}^{*}} \bar{q}(0) e^{-i \omega_{2}^{*} \tau_{2}^{*} \theta}+E_{2},
$$

where $E_{2}=\left(E_{2}^{(1)}, E_{2}^{(2)}, E_{2}^{(3)}\right) \in \mathbb{R}^{3}$.

Next, we seek the appropriate constant vectors $E_{1}$ and $E_{2}$ in expressions (B.17) and (B.19). From equation (B.12) and the definition of $A$, we can have

$$
\int_{-1}^{0} \mathrm{~d} \eta(\theta) W_{20}(\theta)=2 i \omega_{2}^{*} \tau_{2}^{*} W_{20}(0)-H_{20}(0)
$$

$$
\int_{-1}^{0} \mathrm{~d} \eta(\theta) W_{11}(\theta)=-H_{11}(0)
$$

where $\eta(\theta)=\eta(0, \theta)$. Thus,

$$
H_{20}(0)=-g_{20} q(0)-\bar{g}_{02} \bar{q}(0)+2 \tau_{2}^{*}\left(P_{1}, P_{2}, P_{3}\right)^{T},
$$

$$
H_{11}(0)=-g_{11} q(0)-\bar{g}_{11} \bar{q}(0)+\tau_{2}^{*}\left(Q_{1}, Q_{2}, Q_{3}\right)^{T} .
$$

where $P_{1}=-b \beta_{1} q_{3} e^{2 i \omega_{2}^{*} \tau_{1}^{*}}, P_{2}=b \beta_{1} q_{3} e^{-2 i \omega_{2}^{*} \tau_{1}^{*}}-\sigma b \beta_{1} q_{3}-\sigma b$ $\beta_{1} q_{2} q_{3}$, and $P_{3}=-b \beta_{2} q_{2} q_{3} e^{-2 i \omega_{2}^{*} \tau_{2}^{*}}$ and $Q_{1}=-b \beta_{1} \operatorname{Re}\left\{q_{3}\right\}$, $Q_{2}=(1-\sigma) b \beta_{1} \operatorname{Re}\left\{q_{3}\right\}-b \sigma \beta_{1} \operatorname{Re}\left\{q_{2} \bar{q}_{3}\right\}$, and $Q_{3}=-b \beta_{2}$ $\operatorname{Re}\left\{q_{2} \bar{q}_{3}\right\}$.

\section{Note that}

$$
\begin{aligned}
& \left(i \omega_{2}^{*} \tau_{2}^{*} I-\int_{-1}^{0} e^{i \omega_{2}^{*} \tau_{2}^{*} \theta} \mathrm{d} \eta(\theta)\right) q(0)=0, \\
& \left(-i \omega_{2}^{*} \tau_{2}^{*} I-\int_{-1}^{0} e^{-i \omega_{2}^{*} \tau_{2}^{*} \theta} \mathrm{d} \eta(\theta)\right) \bar{q}(0)=0 .
\end{aligned}
$$

Substituting expressions (39) and (B.22) into equation (B.20), we can have

$$
\left(2 i \omega_{2}^{*} \tau_{2}^{*} I-\int_{-1}^{0} e^{2 i \omega_{2}^{*} \tau_{2}^{*} \theta} \mathrm{d} \eta(\theta)\right) E_{1}=2 \tau_{2}^{*}\left(P_{1}, P_{2}, P_{3}\right)^{T},
$$

which leads to $K_{1} E_{1}=2\left(P_{1}, P_{2}, P_{3}\right)^{T}$, where

$$
K_{1}=\left(\begin{array}{ccc}
2 i \omega_{2}^{*}+\mu_{1}+b \beta_{1} V^{*} e^{-2 i \omega_{2}^{*} \tau_{1}^{*}} & 0 & b \beta_{1} S^{*} e^{-2 i \omega_{2}^{*} \tau_{1}^{*}} \\
\left(\sigma-e^{-2 i \omega_{2}^{*} \tau_{1}^{*}}\right) b \beta_{1} V^{*} & 2 i \omega_{2}^{*}+\mu_{1}+\gamma+b \sigma \beta_{1} V^{*} & -b \beta_{1} S^{*} e^{-2 i \omega_{2}^{*} \tau_{1}^{*}}-b \sigma \beta_{1}\left(\frac{\Lambda_{1}}{\mu_{1}}-I^{*}-S^{*}\right) \\
0 & -b \beta_{2}\left(\frac{\Lambda_{2}}{\mu_{2}}-V^{*}\right) e^{-2 i \omega_{2}^{*} \tau_{2}^{*}} & 2 i \omega_{2}^{*}+\mu_{2}+b \beta_{2} I^{*} e^{-2 i \omega_{2}^{*} \tau_{2}^{*}}
\end{array}\right)
$$


It follows that

$$
\begin{aligned}
& E_{1}^{(1)}=\frac{2 \Delta_{11}}{\Delta_{1}}, \\
& E_{1}^{(2)}=\frac{2 \Delta_{12}}{\Delta_{1}}, \\
& E_{1}^{(3)}=\frac{2 \Delta_{13}}{\Delta_{1}},
\end{aligned}
$$

where $\Delta_{1}=\left|K_{1}\right|$,

$$
\begin{aligned}
& \Delta_{11}=\left|\begin{array}{ccc}
P_{1} & 0 & b \beta_{1} S^{*} e^{-2 i \omega_{2}^{*} \tau_{1}^{*}} \\
P_{2} & 2 i \omega_{2}^{*}+\mu_{1}+\gamma+b \sigma \beta_{1} V^{*} & -b \beta_{1} S^{*} e^{-2 i \omega_{2}^{*} \tau_{1}^{*}}-b \sigma \beta_{1}\left(\frac{\Lambda_{1}}{\mu_{1}}-I^{*}-S^{*}\right) \\
P_{3} & -b \beta_{2}\left(\frac{\Lambda_{2}}{\mu_{2}}-V^{*}\right) e^{-2 i \omega_{2}^{*} \tau_{2}^{*}} & 2 i \omega_{2}^{*}+\mu_{2}+b \beta_{2} I^{*} e^{-2 i \omega_{2}^{*} \tau_{2}^{*}}
\end{array}\right|, \\
& \Delta_{12}=\left|\begin{array}{ccc}
2 i \omega_{2}^{*}+\mu_{1}+b \beta_{1} V^{*} e^{-2 i \omega_{2}^{*} \tau_{1}^{*}} & P_{1} & b \beta_{1} S^{*} e^{-2 i \omega_{2}^{*} \tau_{1}^{*}} \\
\left(\sigma-e^{-2 i \omega_{2}^{*} \tau_{1}^{*}}\right) b \beta_{1} V^{*} & P_{2} & -b \beta_{1} S^{*} e^{-2 i \omega_{2}^{*} \tau_{1}^{*}}-b \sigma \beta_{1}\left(\frac{\Lambda_{1}}{\mu_{1}}-I^{*}-S^{*}\right) \\
0 & P_{3} & 2 i \omega_{2}^{*}+\mu_{2}+b \beta_{2} I^{*} e^{-2 i \omega_{2}^{*} \tau_{2}^{*}}
\end{array}\right| \text {, } \\
& \Delta_{13}=\left|\begin{array}{ccc}
2 i \omega_{2}^{*}+\mu_{1}+b \beta_{1} V^{*} e^{-2 i \omega_{2}^{*} \tau_{1}^{*}} & 0 & P_{1} \\
\left(\sigma-e^{-2 i \omega_{2}^{*} \tau_{1}^{*}}\right) b \beta_{1} V^{*} & 2 i \omega_{2}^{*}+\mu_{1}+\gamma+b \sigma \beta_{1} V^{*} & P_{2} \\
0 & -b \beta_{2}\left(\frac{\Lambda_{2}}{\mu_{2}}-V^{*}\right) e^{-2 i \omega_{2}^{*} \tau_{2}^{*}} & P_{3}
\end{array}\right| .
\end{aligned}
$$

Similarly, substituting expressions (B.19) and (B.23) into equation (B.21), we can have

$$
\int_{-1}^{0} \mathrm{~d} \eta(\theta) E_{2}=-\tau_{2}^{*}\left(Q_{1}, Q_{2}, Q_{3}\right)^{T}
$$

which leads to $K_{2} E_{2}=\left(Q_{1}, Q_{2}, Q_{3}\right)^{T}$, where

$$
K_{2}=\left(\begin{array}{ccc}
\mu_{1}+b \beta_{1} V^{*} & 0 & b \beta_{1} S^{*} \\
-(1-\sigma) b \beta_{1} V^{*} & \mu_{1}+\gamma+b \sigma \beta_{1} V^{*} & -\frac{\left(\mu_{1}+\gamma\right) I^{*}}{V^{*}} \\
0 & -b \beta_{2}\left(\frac{\Lambda_{2}}{\mu_{2}}-V^{*}\right) & \mu_{2}+b \beta_{2} I^{*}
\end{array}\right) .
$$

It follows that

$$
\begin{aligned}
& E_{2}^{(1)}=\frac{\Delta_{21}}{\Delta_{2}}, \\
& E_{2}^{(2)}=\frac{\Delta_{22}}{\Delta_{2}}, \\
& E_{2}^{(3)}=\frac{\Delta_{23}}{\Delta_{2}},
\end{aligned}
$$

where $\Delta_{2}=\left|K_{2}\right|$,

$$
\begin{gathered}
\Delta_{21}=\left|\begin{array}{ccc}
Q_{1} & 0 & b \beta_{1} S^{*} \\
Q_{2} & \mu_{1}+\gamma+b \sigma \beta_{1} V^{*} & -\frac{\left(\mu_{1}+\gamma\right) I^{*}}{V^{*}} \\
Q_{3} & -b \beta_{2}\left(\frac{\Lambda_{2}}{\mu_{2}}-V^{*}\right) & \mu_{2}+b \beta_{2} I^{*}
\end{array}\right|, \\
\Delta_{22}=\left|\begin{array}{ccc}
\mu_{1}+b \beta_{1} V^{*} & Q_{1} & b \beta_{1} S^{*} \\
-(1-\sigma) b \beta_{1} V^{*} & Q_{2} & -\frac{\left(\mu_{1}+\gamma\right) I^{*}}{V^{*}} \\
0 & Q_{3} & \mu_{2}+b \beta_{2} I^{*}
\end{array}\right|, \\
\Delta_{23}=\left|\begin{array}{ccc}
\mu_{1}+b \beta_{1} V^{*} & 0 & Q_{1} \\
-(1-\sigma) b \beta_{1} V^{*} & \mu_{1}+\gamma+b \sigma \beta_{1} V^{*} & Q_{2} \\
0 & -b \beta_{2}\left(\frac{\Lambda_{2}}{\mu_{2}}-V^{*}\right) & Q_{3}
\end{array}\right| .
\end{gathered}
$$

\section{Data Availability}

The data included in this paper is only to demonstrate the validity of the results.

\section{Conflicts of Interest}

The authors declare that there is no conflict of interest regarding the publication of this paper.

\section{Acknowledgments}

This work was supported in part by the Fundamental Research Funds for the Central Universities, China (SWU020005), in part by the Natural Science Foundation Project of Chongqing CSTC (cstc2018jcyjAX0810), in part by the Science and Technology Research Program of Chongqing Municipal Education Commission (KJQN201801605), and in part by CQUE (KY201708B and KY20200146).

\section{References}

[1] K. S. Moore, "Vector-borne diseases: an ongoing threat," The journal Nurse Practitioners, vol. 15, no. 6, pp. 449-457, 2019.

[2] F. Catteruccia, "Malaria-carrying mosquitoes get a leg up on insecticides," Nature, vol. 577, no. 7790, pp. 319-320, 2020.

[3] J. Sachs and P. Malaney, "The economic and social burden of malaria," Nature, vol. 415, no. 6872, pp. 680-685, 2002.

[4] H.-M. Wei, X.-Z. Li, and M. Martcheva, "An epidemic model of a vector-borne disease with direct transmission and time delay," Journal of Mathematical Analysis and Application, vol. 342, no. 2, pp. 895-908, 2008.

[5] J. Xu and Y. Zhou, "Hopf bifurcation and its stability for a vector-borne disease model with delay and reinfection," Applied Mathematical Modeling, vol. 40, no. 3, pp. 16851702, 2016.

[6] Z. Hu, S. Yin, and H. Wang, "Stability and Hopf bifurcation of a vector-borne disease model with saturated infection rate and 
reinfection," Computational and Mathematical Methods in Medicine, vol. 2019, Article ID 1352698, 17 pages, 2019.

[7] A. M. Niger and A. B. Gumel, "Mathematical analysis of the role of repeated exposure on malaria transmission dynamics," Differential Equations and Dynamical Systems, vol. 16, no. 3, pp. 251-287, 2008.

[8] L. Hviid, "Naturally acquired immunity to Plasmodium falciparum malaria in Africa," Acta Tropica, vol. 95, no. 3, pp. 270-275, 2005.

[9] Y. Xue and T. Li, "Stability and Hopf bifurcation for a delayed SIR epidemic model with logistic growth," Abstract and Applied Analysis, vol. 2013, Article ID 916130, 11 pages, 2013.

[10] Z. Zhang, S. Kundu, J. P. Tripathi, and S. Bugalia, "Stability and Hopf bifurcation analysis of an SVEIR epidemic model with vaccination and multiple time delays," Chaos Solitons \& Fractals, vol. 131, article 109483, 2020.

[11] H. Wan and J. A. Cui, "A malaria model with two delays," Discrete Dynamics in Nature and Society, vol. 2013, Article ID 601265, 8 pages, 2013.

[12] J. L. Liu and T. L. Zhang, "Stability and Hopf bifurcation analysis of a plant virus propagation model with two delays," Discrete Dynamics in Nature and Society, vol. 2018, Article ID 7126135, 12 pages, 2018.

[13] L. Li, C. H. Zhang, and X. P. Yan, "Stability and Hopf bifurcation analysis for a two-enterprise interaction model with delays," Communications in Nonlinear Science and Numerical Simulation, vol. 30, no. 1-3, pp. 70-83, 2016.

[14] C. Xu and M. Liao, "Bifurcation analysis of an autonomous epidemic predator-prey model with delay," Annali di Matematica Pura ed Applicata, vol. 193, no. 1, pp. 23-38, 2014.

[15] C. $\mathrm{Xu}$ and P. Li, "Oscillations for a delayed predator-prey model with Hassell-Varley-type functional response," Comptes Rendus Biologies, vol. 338, no. 4, pp. 227-240, 2015.

[16] S. Khajanchi, M. Perc, and D. Ghosh, "The influence of time delay in a chaotic cancer model," Chaos, vol. 28, article 103101, 2018.

[17] C. Xu, M. Liao, and P. Li, "Bifurcation control of a fractionalorder delayed competition and cooperation model of two enterprises," Science China Technological Sciences, vol. 62, no. 12, pp. 2130-2143, 2019.

[18] R. Kumar, A. K. Sharma, and K. Agnihotri, "Hopf bifurcation analysis in a multiple delayed innovation diffusion model with Holling II functional response," Mathematical Methods in the Applied Sciences, vol. 43, no. 4, pp. 2056-2075, 2020.

[19] Z. Zhang and H. Yang, "Stability and bifurcation in a stagestructured predator-prey system with Holling-II functional response and multiple delays," International Journal of Computer Mathematics, vol. 92, no. 3, pp. 542-561, 2015.

[20] B. D. Hassard, N. D. Kazarinoff, and Y. H. Wan, "Theory and applications of Hopf bifurcation," London Mathematical Society Lecture Note Series, vol. 41, 1981.

[21] C. Castillochavez and H. R. Thieme, "Asymptotically autonomous epidemic models," in Mathematical Population Dynamics: Analysis of Heterogeneity, Theory of Epidemics, vol. 1, Wuerz, Winnipeg, Canada, 1994.

[22] M. Jackson and B. M. Chen-Charpentier, "Modeling plant virus propagation with delays," Journal of Computational and Applied Mathematics, vol. 309, no. 47, pp. 611-621, 2017.

[23] P. Driessche and J. Watmough, "Further notes on the basic reproduction number," in Mathematical Epidemiology Lecture Notes in Mathematics, vol. 1945, Springer, Berlin, 2008.
[24] J. M. Heffernan, R. J. Smith, and L. M. Wahl, "Perspectives on the basic reproductive ratio," Journal of the Royal Society Interface, vol. 2, no. 4, pp. 281-293, 2005.

[25] Y. Song and S. Yuan, "Bifurcation analysis in a predator-prey system with time delay," Nonlinear Analysis: Real World Applications, vol. 7, no. 2, pp. 265-284, 2006.

[26] Y. Song and J. Wei, "Bifurcation analysis for Chen's system with delayed feedback and its application to control of chaos," Chaos, Solitons \& Fractals, vol. 22, no. 1, pp. 75-91, 2004.

[27] J. Hale, "Theory and application of Hopf bifurcation," in London Math Society Lecture Note Series, Cambridge University Press, Cambridge, UK, 1977. 